$\mathbb{T}$ periodica polytechnica

Civil Engineering

$51 / 2(2007) 2335$

doi: 10.3311/pp.ci.2007-2.05

web: http://www.pp.bme.hu/ci

(c) Periodica Polytechnica 2007

RESEARCH ARTICLE

\section{BEM formulation for plane orthotropic bodies - a modification for exterior regions and its proof}

\author{
György Szeidl / Judit Dudra
}

Received 2007-11-07

\begin{abstract}
Assuming linear displacements and constant strains and stresses at infinity, our aim is to reformulate the equations of the direct boundary element method for plane problems of elasticity. We shall consider a body made of orthotropic material. The equations we have reformulated make possible to attack plane problems on exterior regions without replacing the region in question by a bounded one.
\end{abstract}

\section{Keywords}

Boundary element method - Somigliana relations - direct method $\cdot$ orthotropic body $\cdot$ plane problems $\cdot$ exterior regions

\section{Acknowledgement}

The support provided by the Hungarian National Research Foundation within the framework of the project OTKA T046834 is gratefully acknowledged.

\section{György Szeidl}

Department of Mechanics, University of Miskolc,, H-3515 MiskolcEgyetemváros,, Hungary

e-mail: Gyorgy.SZEIDL@ uni-miskolc.hu

\section{Judit Dudra}

Department of Mechanics, University of Miskolc,, H-3515 MiskolcEgyetemváros,, Hungary

e-mail: judit.dudra@gmail.com

\section{Introduction}

As is well known a large literature studies plane problems for orthotropic bodies, including for instance [9], [8], [7] as well as the books [1,2] and the references therein. However, as explained by Schiavone [10], the standard formulation for exterior regions has the disadvantage that it is impossible to prescribe a constant stress state at infinity.

The reason is that an assumption about the far field pattern of the displacements is needed in order to establish an appropriate Betti formula and to prove uniqueness and existence for the exterior Dirichlet and Neuman problems. Unfortunately, this assumption excludes those problems from the theory for which the displacements are linear while the strains and stresses are constant at infinity.

To make progress on plane problems with such displacements, we note that if the direct formulation reproduces this displacement field, then the resulting strain and stress conditions must also be constant at infinity. Consequently, plane problems for the exterior regions can be attacked without replacing the region by a bounded one. The work in [11] and [12] presents such direct formulations by assuming constant strains and stresses at infinity for an isotropic body. For exterior regions [11] reformulates the classical approach to plane problems. For the same class of problems but in a dual formulation [12] sets up the equations of the direct method in terms of stress functions of order one.

The present paper is an attempt to clarify how the formulation changes if we apply the ideas presented in paper [11] to orthotropic bodies.

\section{Preliminaries}

Throughout this paper $x_{1}$ and $x_{2}$ are rectangular Cartesian coordinates, referred to an origin $O$. Greek subscripts are assumed to have the range $(1,2)$, summation over repeated subscripts is implied. The doubly connected exterior region under consideration is denoted by $A_{e}$ and is bounded by the contour $\mathcal{L}_{o}$. We stipulate that the contour admits a nonsingular parametrization in terms of its arc length $s$. The outer normal is denoted by $n_{\pi}$. In accordance with the notations introduced, $\delta_{\kappa \lambda}$ is the Kronecker 
symbol, $\partial_{\alpha}$ stands for the derivatives taken with respect to $x_{\alpha}$ and $\epsilon_{3 \kappa \lambda}$ is the permutation symbol. Assuming plane problems let $u_{\kappa}, e_{\kappa \lambda}$ and $t_{\kappa \lambda}$ be the displacement field and the in plane components of strain and stress, respectively. For orthotropic bodies the material constants are denoted by $s_{11}, s_{12}=s_{21}, s_{22}$ and $s_{66}$.

For homogenous and orthotropic material the plane problem of classical elasticity is governed by the kinematic equations

$$
e_{\rho \lambda}=\frac{1}{2}\left(\partial_{\rho} u_{\lambda}+\partial_{\lambda} u_{\rho}\right)
$$

Hooke's law

$$
\begin{aligned}
& t_{11}=c_{11} e_{11}+c_{12} e_{22}, \\
& t_{22}=c_{12} e_{11}+c_{22} e_{22}, \\
& t_{12}=t_{21}=2 c_{66} e_{12},
\end{aligned}
$$

where

$$
\begin{array}{r}
c_{11}=\frac{s_{22}}{d}, \quad c_{12}=c_{21}=-\frac{s_{12}}{d}, \quad c_{22}=\frac{s_{11}}{d}, \\
c_{66}=\frac{1}{s_{66}}, \quad d=s_{11} s_{22}-s_{12}^{2} ;
\end{array}
$$

and the equilibrium equations

$$
t_{\rho \lambda} \partial_{\lambda}+b_{\rho}=0
$$

which should be complemented with appropriate boundary conditions not detailed here since they play no role in the present investigations. The basic equation for $u_{\lambda}$ takes the form

$$
\mathcal{D}_{\rho \lambda} u_{\lambda}+b_{\rho}=0
$$

where the differential operator $\mathcal{D}_{\rho \lambda}$ has the form

$$
\left[\mathcal{D}_{\rho \lambda}\right]=\left[\begin{array}{cc}
c_{11} \partial_{1}^{2}+c_{66} \partial_{2}^{2} & \left(c_{12}+c_{66}\right) \partial_{1} \partial_{2} \\
\left(c_{21}+c_{66}\right) \partial_{2} \partial_{1} & c_{22} \partial_{2}^{2}+c_{66} \partial_{1}^{2}
\end{array}\right]
$$

Let $Q\left(\xi_{1}, \xi_{2}\right)$ and $M\left(x_{1}, x_{2}\right)$ be two points in the plane (the source point and the field point). We shall assume temporarily that the point $Q$ is fixed. The distance between $Q$ and $M$ is $R$, the position vector of $M$ relative to $Q$ is $r_{\kappa}$. The small circle as a subscript (for instance $M_{\circ}$ or $Q_{\circ}$ ) indicates that the corresponding points, i.e., $Q$ or $M$ are taken on the contour.

It is obvious that

$$
r_{\alpha}(M, Q)=x_{\alpha}(M)-\xi_{\alpha}(Q)=x_{\alpha}-\xi_{\alpha}
$$

Let us introduce the following notations

$$
\begin{aligned}
\lambda_{1}+\lambda_{2} & =\left(2 s_{12}+s_{66}\right) / s_{22}, \\
\lambda_{1} \lambda_{2} & =s_{11} / s_{22}, \\
A_{\alpha} & =s_{12}-\lambda_{\alpha} s_{22}, \\
\rho_{\alpha}^{2} & =\lambda_{\alpha} r_{1}^{2}+r_{2}^{2}, \\
D & =\frac{1}{2 \pi\left(\lambda_{1}-\lambda_{2}\right) s_{22}} .
\end{aligned}
$$

For our later considerations we note that Eqs. (7) and (8) imply

$$
\lambda_{1,2}=\frac{2 s_{21}+s_{66}}{2 s_{22}} \pm \sqrt{\left(\frac{2 s_{21}+s_{66}}{2 s_{22}}\right)^{2}-\frac{s_{11}}{s_{22}}} .
$$

The well-known singular fundamental solutions for the basic Eq. (5a) [7, 9] are given by the formulas

$$
\begin{aligned}
& U_{11}(M, Q)=D\left(\sqrt{\lambda_{1}} A_{2}^{2} \ln \rho_{1}-\sqrt{\lambda_{2}} A_{1}^{2} \ln \rho_{2}\right), \\
& U_{12}(M, Q)=D A_{1} A_{2} \arctan \frac{\left(\sqrt{\lambda_{1}}-\sqrt{\lambda_{2}}\right) r_{1} r_{2}}{\sqrt{\lambda_{1}} \sqrt{\lambda_{2}} r_{1}^{2}+r_{2}^{2}}, \\
& U_{21}(M, Q)=U_{12}(M, Q), \\
& U_{22}(M, Q)=-D\left(\frac{A_{1}^{2} \ln \rho_{1}}{\sqrt{\lambda_{1}}}-\frac{A_{2}^{2} \ln \rho_{2}}{\sqrt{\lambda_{2}}}\right)
\end{aligned}
$$

and

$$
\begin{aligned}
& T_{11}(M, Q)=D\left[\frac{\sqrt{\lambda_{2}} A_{1}}{\rho_{2}^{2}}-\frac{\sqrt{\lambda_{1}} A_{2}}{\rho_{1}^{2}}\right]\left(r_{1} n_{1}+r_{2} n_{2}\right), \\
& T_{12}(M, Q)=D\left\{\left(\frac{\sqrt{\lambda_{1}} A_{1}}{\rho_{1}^{2}}-\frac{\sqrt{\lambda_{2}} A_{2}}{\rho_{2}^{2}}\right) r_{1} n_{2}-\right. \\
& \left.\left(\frac{1}{\sqrt{\lambda_{1}}} \frac{A_{1}}{\rho_{1}^{2}}-\frac{1}{\sqrt{\lambda_{2}}} \frac{A_{2}}{\rho_{2}^{2}}\right) r_{2} n_{1}\right\} \text {, } \\
& T_{21}(M, Q)=D\left\{\left(\frac{\lambda_{1} \sqrt{\lambda_{1}} A_{2}}{\rho_{1}^{2}}-\frac{\lambda_{2} \sqrt{\lambda_{2}} A_{1}}{\rho_{2}^{2}}\right) r_{1} n_{2}-\right. \\
& \left.\left(\frac{\sqrt{\lambda_{1}} A_{2}}{\rho_{1}^{2}}-\frac{\sqrt{\lambda_{2}} A_{1}}{\rho_{2}^{2}}\right) r_{2} n_{1}\right\} \text {, } \\
& T_{22}(M, Q)=D\left[\frac{\sqrt{\lambda_{1}} A_{1}}{\rho_{1}^{2}}-\frac{\sqrt{\lambda_{2}} A_{2}}{\rho_{2}^{2}}\right]\left(r_{1} n_{1}+r_{2} n_{2}\right) \text {, }
\end{aligned}
$$

where

$$
\mathfrak{u}_{\lambda}(M)=U_{\lambda \kappa}(M, Q) e_{\kappa}(Q), \text { and } t_{\lambda}(M)=T_{\lambda \kappa}(M, Q) e_{\kappa}(Q)
$$

are the displacement vector and stress vector on a line element with a normal $n_{\lambda}=n_{\lambda}(M)$ to it at the point $M$ due to the force $e_{\kappa}=e_{\kappa}(Q)$ at $Q$.

\section{Basic formulas for exterior regions}

Fig. 1 depicts a triple connected region $A_{e}^{\prime}$ bounded by the contours $\mathcal{L}_{o}, \mathcal{L}_{\varepsilon}$ and the circle $\mathcal{L}_{R}$ with radius ${ }_{e} R$ and center at $O$. Here $\mathcal{L}_{\varepsilon}$ is the contour of the neighborhood $A_{\varepsilon}$ of $Q$ with radius $R_{\varepsilon}$ while ${ }_{e} R$ is sufficiently large so that the region bounded by $\mathcal{L}_{R}$ covers both $\mathcal{L}_{0}$, and $\mathcal{L}_{\varepsilon}$. If ${ }_{e} R \rightarrow \infty$ and $R_{\varepsilon} \rightarrow 0$ then clearly $A_{e}^{\prime} \rightarrow A_{e}$.

Let $u_{\kappa}(M)$ and $g_{\kappa}(M)$ be sufficiently smooth - continuously differentiable at least twice - but otherwise arbitrary displacement fields on $A_{e}$. The stresses obtained from these displacement fields are denoted by $t_{\lambda \kappa}\left[u_{\rho}(M)\right]$ and $t_{\lambda \kappa}\left[g_{\rho}(M)\right]$ respectively. 


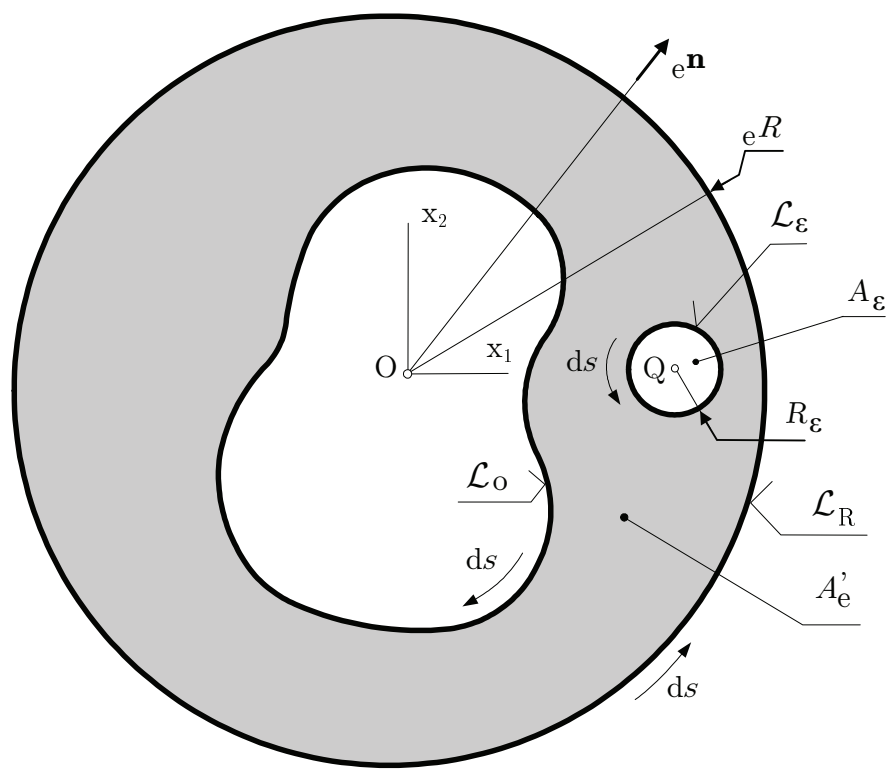

Fig. 1.

Equation

$\int_{A_{e}^{\prime}}\left[u_{\lambda}(M)\left(\mu \stackrel{M}{\mathcal{D}}{ }_{\lambda \sigma} g_{\sigma}(M)\right)-g_{\lambda}(M)\left(\mu \stackrel{M}{\mathcal{D}} \lambda_{\sigma} u_{\sigma}(M)\right)\right] \mathrm{d} A_{M}=$

$\oint_{\mathcal{L}_{o}}\left[u_{\lambda}\left(M_{\circ}\right) t_{\lambda \kappa}\left[g_{\rho}\left(M_{\circ}\right)\right] n_{\kappa}\left(M_{\circ}\right)-g_{\lambda}\left(M_{\circ}\right) t_{\lambda \kappa}\left[u_{\rho}\left(M_{\circ}\right)\right] n_{\kappa}\left(M_{\circ}\right)\right] \mathrm{d} s_{M_{\circ}}+$

$\oint_{\mathcal{L}_{\varepsilon}}\left[u_{\lambda}\left(M_{\circ}\right) t_{\lambda \kappa}\left[g_{\rho}\left(M_{\circ}\right)\right] n_{\kappa}\left(M_{\circ}\right)-g_{\lambda}\left(M_{\circ}\right) t_{\lambda \kappa}\left[u_{\rho}\left(M_{\circ}\right)\right] n_{\kappa}\left(M_{\circ}\right)\right] \mathrm{d} s_{M_{\circ}}+$

$\oint_{\mathcal{L}_{R}}\left[u_{\lambda}\left(M_{\circ}\right) t_{\lambda \kappa}\left[g_{\rho}\left(M_{\circ}\right)\right] n_{\kappa}\left(M_{\circ}\right)-g_{\lambda}\left(M_{\circ}\right) t_{\lambda \kappa}\left[u_{\rho}\left(M_{\circ}\right)\right] n_{\kappa}\left(M_{\circ}\right)\right] \mathrm{d} s_{M_{\circ}}$,

in which $M$ over a letter denotes derivatives taken with respect to the point coordinates $M$ and $n_{\kappa}\left(M_{\circ}\right)$ is the outward normal, is the primal Somigliana identity applied to the triple connected region $A_{e}^{\prime}$.

Let $g_{\lambda}(Q)=U_{\lambda \kappa}(M, Q) e_{\kappa}(Q)$, which is a non singular elastic state of the plane in $A_{e}^{\prime}$. We regard $u_{\lambda}(M)$ as a different elastic state in the region $A_{e}$. Further we assume that $u_{\lambda}(M)$ has the far field pattern (asymptotic behaviour)

$$
\tilde{u}_{\kappa}(M)=c_{\kappa}+\varepsilon_{3 \rho \kappa} x_{\rho} \omega+e_{\kappa \beta}(\infty) x_{\beta}
$$

as $x_{\beta}$ or equivalently $M$ tends to infinity. Here $c_{\kappa}$ is a translation, $\omega$ is a rotation in finite, $c_{\kappa}+\varepsilon_{3 \rho \kappa} x_{\rho} \omega$ is the corresponding rigid body motion, $e_{\kappa \beta}(\infty)$ is a constant strain tensor at infinity and $e_{\kappa \beta}(\infty) x_{\beta}$ is the corresponding displacement field.

The stresses induced by the strains $e_{\kappa \beta}(\infty)$ can be obtained by the Hooke law:

$$
\begin{aligned}
& t_{11}(\infty)=c_{11} e_{11}(\infty)+c_{12} e_{22}(\infty) \\
& t_{22}(\infty)=c_{12} e_{11}(\infty)+c_{22} e_{22}(\infty) \\
& t_{12}(\infty)=t_{21}(\infty)=2 c_{66} e_{12}(\infty)
\end{aligned}
$$

Substituting the above quantities into the Somigliana identity, we obtain

$$
\begin{aligned}
\int_{A_{e}^{\prime}}\left[u_{\lambda}(M)\left(\mu \stackrel{M}{\mathcal{D}}{ }_{\lambda \sigma} U_{\sigma \kappa}(M, Q)\right)-\right. \\
\left.\quad\left(\mu \stackrel{M}{\mathcal{D}}{ }_{\lambda \sigma} u_{\sigma}(M)\right) U_{\lambda \kappa}(M, Q)\right] \mathrm{d} A_{M} e_{\kappa}(Q)= \\
=\oint_{\mathcal{L}_{o}}\left[u_{\lambda}\left(M_{\circ}\right) T_{\lambda \kappa}\left(M_{\circ}, Q\right)-t_{\lambda}\left(M_{\circ}\right) U_{\lambda \kappa}\left(M_{\circ}, Q\right)\right] \mathrm{d} s_{M_{\circ}} e_{K}(Q)+ \\
\oint_{\mathcal{L}_{\varepsilon}}\left[u_{\lambda}\left(M_{\circ}\right) T_{\lambda \kappa}\left(M_{\circ}, Q\right)-t_{\lambda}\left(M_{\circ}\right) U_{\lambda \kappa}\left(M_{\circ}, Q\right)\right] \mathrm{d} s_{M_{\circ}} e_{\kappa}(Q) \\
\oint_{\mathcal{L}_{R}}\left[u_{\lambda}\left(M_{\circ}\right) T_{\lambda \kappa}\left(M_{\circ}, Q\right)-t_{\lambda}\left(M_{\circ}\right) U_{\lambda \kappa}\left(M_{\circ}, Q\right)\right] \mathrm{d} s_{M_{\circ}} e_{\kappa}(Q)
\end{aligned}
$$

since $t_{\lambda \kappa}\left[u_{\rho}\left(M_{\circ}\right)\right] n_{\kappa}\left(M_{\circ}\right)=t_{\lambda}\left(M_{\circ}\right)$ is the stress on the contour and obviously

$$
t_{\lambda \kappa}\left[g_{\rho}\left(M_{\circ}\right)\right] n_{\kappa}\left(M_{\circ}\right)=T_{\lambda \kappa}\left(M_{\circ}, Q\right) e_{\kappa}(Q) .
$$

In the sequel we shall assume that there are no body forces. This assumption has no effect on the result we will obtain.

It is clear that one can omit $e_{\kappa}(Q)$. Regarding the equation obtained by omitting $e_{\kappa}(Q)$, our goal is to compute its limit as $R_{\varepsilon} \rightarrow 0$ and ${ }_{e} R \rightarrow \infty$. As is well known, the left hand side vanishes under the conditions detailed above(see [1] ), and hence

$\oint_{\mathcal{L}_{o}} \cdots+\lim _{R_{\varepsilon} \rightarrow 0} \oint_{\mathcal{L}_{\varepsilon}} \cdots=$

$u_{\kappa}(Q)+\oint_{\mathcal{L}_{o}}\left[u_{\lambda}(\stackrel{o}{M}) T_{\lambda \kappa}\left(M_{\circ}, Q\right)-t_{\lambda}\left(M_{\circ}\right) U_{\lambda \kappa}\left(M_{\circ}, Q\right)\right] \mathrm{d} s_{M_{\circ}}$.

Consequently

$$
\begin{aligned}
& u_{\kappa}(Q)= \\
& \lim _{e R \rightarrow \infty} \oint_{\mathcal{L}_{R}}\left[t_{\lambda}\left(M_{\circ}\right) U_{\lambda \kappa}\left(M_{\circ}, Q\right)-u_{\lambda}\left(M_{\circ}\right) T_{\lambda \kappa}\left(M_{\circ}, Q\right)\right] \mathrm{d} s_{M_{\circ}}+ \\
& \oint_{\mathcal{L}_{o}}\left[t_{\lambda}\left(M_{\circ}\right) U_{\lambda \kappa}\left(M_{\circ}, Q\right)-u_{\lambda}\left(M_{\circ}\right) T_{\lambda \kappa}\left(M_{\circ}, Q\right)\right] \mathrm{d} s_{M_{\circ}} .
\end{aligned}
$$

In order to establish the first Somigliana formula for the exterior region, we need to find the limit of the first integral on the right hand side.

\section{Somigliana formulas modified for exterior regions}

In this section our main objective is to prove that

$$
\begin{gathered}
I_{\kappa}= \\
\lim _{e R \rightarrow \infty} \oint_{\mathcal{L}_{R}}\left[t_{\lambda}\left(M_{\circ}\right) U_{\lambda \kappa}\left(M_{\circ}, Q\right)-u_{\lambda}\left(M_{\circ}\right) T_{\lambda \kappa}\left(M_{\circ}, Q\right)\right] \mathrm{d} s_{M_{\circ}}= \\
c_{\kappa}+\varepsilon_{3 \rho \kappa} \xi_{\rho} \omega+e_{\kappa \beta}(\infty) \xi_{\beta}=\tilde{u}_{\kappa}(Q) .
\end{gathered}
$$

Using

$$
\left.\left.x_{\beta} \stackrel{o}{M}\right)={ }_{e} R n_{\beta} \stackrel{o}{M}\right)
$$


we substitute $t_{\lambda \rho}(\infty) n_{\rho}\left(M_{\circ}\right)$ for $t_{\lambda}\left(M_{\circ}\right)$ and $c_{\lambda}+\varepsilon_{3 \rho \lambda} x_{\rho} \omega+$ $e_{\kappa \beta}(\infty) x_{\beta}$ for $u_{\lambda}\left(M_{\circ}\right)$. This implies

$$
\begin{aligned}
I_{\kappa}= & \stackrel{(1)}{I_{\kappa}}+\stackrel{(2)}{I_{\kappa}}+\stackrel{(3)}{I_{\kappa}}+\stackrel{(4)}{I_{\kappa}}= \\
& -\lim _{e R \rightarrow \infty} \oint_{\mathcal{L}_{R}} c_{\lambda} T_{\lambda \kappa}\left(M_{\circ}, Q\right) \mathrm{d} s_{M_{\circ}}- \\
& \lim _{e} \varepsilon_{3 \rho \lambda e} R \omega \oint_{\mathcal{L}_{R}} n_{\rho}(\stackrel{o}{M}) T_{\lambda \kappa}\left(M_{\circ}, Q\right) \mathrm{d} s_{M_{\circ}}+ \\
& \lim _{e} t_{\lambda \rightarrow \infty}(\infty) \oint_{\mathcal{L}_{R}} n_{\rho}\left(M_{\circ}\right) U_{\lambda \kappa}\left(M_{\circ}, Q\right) \mathrm{d} s_{M_{\circ}}- \\
& \lim _{e} e_{\lambda \rightarrow \infty}(\infty)_{e} R \oint_{\mathcal{L}_{R}} n_{\beta}(\stackrel{o}{M}) T_{\lambda \kappa}\left(M_{\circ}, Q\right) \mathrm{d} s_{M_{\circ}} .
\end{aligned}
$$

REMARK 1.: In accordance with Eq. 16, the stresses and strains are taken as constant quantities which are therefore independent of the arc coordinate $s$.

Since

$$
\oint_{\mathcal{L}_{R}} T_{\lambda \kappa}\left(M_{\circ}, Q\right) \mathrm{d} s_{M_{\circ}}=-\delta_{\kappa \lambda}
$$

and

$$
\varepsilon_{3 \rho \lambda} \oint_{\mathcal{L}_{R}} r_{\rho} T_{\lambda \kappa}\left(M_{\circ}, Q\right) \mathrm{d} s_{M_{\circ}}=0
$$

which is the moment about the origin of the stresses due to a unit force applied at $Q$, one can write

$$
\begin{gathered}
\stackrel{(1)}{I_{\kappa}}+\stackrel{(2)}{I_{\kappa}}=c_{\kappa}-\lim _{e} \varepsilon_{3 \rho \lambda} \omega \oint_{\mathcal{L}_{R}}\left(\xi_{\rho}+r_{\rho}\right) T_{\lambda \kappa}\left(M_{\circ}, Q\right) \mathrm{d} s_{M_{\circ}}= \\
c_{\kappa}-\lim _{e R \rightarrow \infty}\left[\varepsilon_{3 \rho \lambda} \omega \xi_{\rho} \oint_{\mathcal{L}_{R}} T_{\lambda \kappa}\left(M_{\circ}, Q\right) \mathrm{d} s_{M_{\circ}}+\right. \\
\left.\varepsilon_{3 \rho \lambda} \omega \oint_{\mathcal{L}_{R}} r_{\rho} T_{\lambda \kappa}\left(M_{\circ}, Q\right) \mathrm{d} s_{M_{\circ}}\right]=c_{\kappa}+\varepsilon_{3 \rho \kappa} \omega \xi_{\rho}
\end{gathered}
$$

which clearly shows that (22) reproduces the rigid body motion.

Determining the limits $\stackrel{(3)}{I}_{\kappa}$ and $\stackrel{(4)}{I}_{\kappa}$ requires long formal transformations. For this reason we confine ourselves to basic argument and the results of the most important steps.

First, we have to expand $U_{\lambda \kappa}$ and $T_{\lambda \kappa}$ into series in terms of ${ }_{e} R$ to the power $1,0,-1,-2$ etc. These transformations are based on the relations:

$$
\begin{gathered}
n_{\alpha}(\stackrel{o}{M})=n_{\alpha} \\
r_{\alpha}=x_{\alpha}(\stackrel{o}{M})-\xi_{\alpha}(Q)=x_{\alpha}-\xi_{\alpha}=e_{e} R\left(n_{\alpha}-\frac{\xi_{\alpha}}{e R}\right) \\
\rho_{\alpha}=\sqrt{\lambda_{\alpha} r_{1}^{2}+r_{2}^{2}} \simeq \\
\simeq e_{e} R \sqrt{\lambda_{\alpha} n_{1}^{2}+n_{2}^{2}}\left\{1-\frac{1}{e R} \frac{\lambda_{\alpha} n_{1} \xi_{1}+n_{2} \xi_{2}}{\lambda_{\alpha} n_{1}^{2}+n_{2}^{2}}+\frac{1}{2_{e} R^{2}} \frac{\lambda_{\alpha} \xi_{1}^{2}+\xi_{2}^{2}}{\lambda_{\alpha} n_{1}^{2}+n_{2}^{2}}\right\} \\
\frac{1}{\ln \rho_{\alpha} \simeq \ln _{e} R+\frac{1}{2} \ln \left(\lambda_{\alpha} n_{1}^{2}+n_{2}^{2}\right)-} \\
\frac{1}{e R} \frac{\lambda_{\alpha} n_{1} \xi_{1}+n_{2} \xi_{2}}{\lambda_{\alpha} n_{1}^{2}+n_{2}^{2}}+\frac{1}{e_{e} R^{2}} \frac{\lambda_{\alpha} \xi_{1}^{2}+\xi_{2}^{2}}{\lambda_{\alpha} n_{1}^{2}+n_{2}^{2}}, \\
\frac{1}{\rho_{\alpha}^{2}}\left(1+\frac{2}{e R} \frac{\lambda_{\alpha} n_{1} \xi_{1}+n_{2} \xi_{2}}{\lambda_{\alpha} n_{1}^{2}+n_{2}^{2}}-\frac{1}{e R^{2}} \frac{\lambda_{\alpha} \xi_{1}^{2}-\xi_{2}^{2}}{\lambda_{\alpha} n_{1}^{2}+n_{2}^{2}}\right)
\end{gathered}
$$

and

$$
\arctan (x+\varepsilon a)=\arctan x+\frac{\varepsilon a}{1+x^{2}},
$$

where $a$ is constant and $\varepsilon$ is a very small quantity.

Consequently

$$
\begin{aligned}
& \arctan \frac{\left(\sqrt{\lambda_{1}}-\sqrt{\lambda_{2}}\right) r_{1} r_{2}}{\sqrt{\lambda_{1}} \sqrt{\lambda_{2}} r_{1}^{2}+r_{2}^{2}} \simeq \arctan \frac{\left(\sqrt{\lambda_{1}}-\sqrt{\lambda_{2}}\right) n_{1} n_{2}}{\sqrt{\lambda_{1} \lambda_{2}} n_{1}^{2}+n_{2}^{2}}+ \\
& \frac{\left(\sqrt{\lambda_{1}}-\sqrt{\lambda_{2}}\right)}{e R} \frac{\left(\sqrt{\lambda_{1} \lambda_{2}} n_{1}^{2} n_{2}-n_{2}^{3}\right) \xi_{1}-\left(\sqrt{\lambda_{1} \lambda_{2}} n_{1}^{3}-n_{1} n_{2}^{2}\right) \xi_{2}}{n_{1}^{4} \lambda_{1} \lambda_{2}+n_{2}^{4}+\left(\lambda_{1}+\lambda_{2}\right) n_{1}^{2} n_{2}^{2}} .
\end{aligned}
$$

Using relations 23a, ..., 23f for a sufficiently large ${ }_{e} R$ we have

$$
\begin{aligned}
& U_{11}\left(M_{\circ}, Q\right) \simeq \\
& D\left[\sqrt{\lambda_{1}} A_{2}^{2}\left(\ln _{e} R+\frac{1}{2} \ln \left(\lambda_{1} n_{1}^{2}+n_{2}^{2}\right)-\frac{1}{e^{R}} \frac{\lambda_{1} n_{1} \xi_{1}+n_{2} \xi_{2}}{\lambda_{1} n_{1}^{2}+n_{2}^{2}}\right)-\right. \\
& \left.\quad \sqrt{\lambda_{2}} A_{1}^{2}\left(\ln _{e} R+\frac{1}{2} \ln \left(\lambda_{2} n_{1}^{2}+n_{2}^{2}\right)-\frac{1}{e^{R}} \frac{\lambda_{2} n_{1} \xi_{1}+n_{2} \xi_{2}}{\lambda_{2} n_{1}^{2}+n_{2}^{2}}\right)\right],
\end{aligned}
$$

$$
\begin{aligned}
& U_{22}\left(M_{\circ}, Q\right) \simeq \\
& -D\left[\frac{A_{1}^{2}}{\sqrt{\lambda_{1}}}\left(\ln _{e} R+\frac{1}{2} \ln \left(\lambda_{1} n_{1}^{2}+n_{2}^{2}\right)-\frac{1}{e^{R}} \frac{\lambda_{1} n_{1} \xi_{1}+n_{2} \xi_{2}}{\lambda_{1} n_{1}^{2}+n_{2}^{2}}\right)-\right. \\
& \left.\frac{A_{2}^{2}}{\sqrt{\lambda_{2}}}\left(\ln _{e} R+\frac{1}{2} \ln \left(\lambda_{\alpha} n_{1}^{2}+n_{2}^{2}\right)-\frac{1}{e^{R}} \frac{\lambda_{\alpha} n_{1} \xi_{1}+n_{2} \xi_{2}}{\lambda_{\alpha} n_{1}^{2}+n_{2}^{2}}\right)\right],
\end{aligned}
$$

$$
\begin{aligned}
U_{12}\left(M_{\circ}, Q\right) \simeq & D A_{1} A_{2}\left[\arctan \frac{\left(\sqrt{\lambda_{1}}-\sqrt{\lambda_{2}}\right) n_{1} n_{2}}{\sqrt{\lambda_{1} \lambda_{2}} n_{1}^{2}+n_{2}^{2}}+\frac{\left(\sqrt{\lambda_{1}}-\sqrt{\lambda_{2}}\right)}{e^{R}} \times\right. \\
& \left.\frac{\left(\sqrt{\lambda_{1} \lambda_{2}} n_{1}^{2} n_{2}-n_{2}^{3}\right) \xi_{1}-\left(\sqrt{\lambda_{1} \lambda_{2}} n_{1}^{3}-n_{1} n_{2}^{2}\right) \xi_{2}}{n_{1}^{4} \lambda_{1} \lambda_{2}+n_{2}^{4}+\left(\lambda_{1}+\lambda_{2}\right) n_{1}^{2} n_{2}^{2}}\right]
\end{aligned}
$$

and

$$
\begin{aligned}
& T_{11}\left(M_{\circ}, Q\right)= \\
& \quad \frac{D}{e^{R}} \frac{\sqrt{\lambda_{2}} A_{1}}{\lambda_{2} n_{1}^{2}+n_{2}^{2}}\left[1+\frac{2}{e^{R}} \frac{\lambda_{2} n_{1} \xi_{1}+n_{2} \xi_{2}}{\lambda_{2} n_{1}^{2}+n_{2}^{2}}-\frac{1}{e^{R}}\left(\xi_{1} n_{1}+\xi_{2} n_{2}\right)\right]- \\
& -\frac{D}{e^{R}} \frac{\sqrt{\lambda_{1}} A_{2}}{\lambda_{1} n_{1}^{2}+n_{2}^{2}}\left[1+\frac{2}{e^{R}} \frac{\lambda_{1} n_{1} \xi_{1}+n_{2} \xi_{2}}{\lambda_{1} n_{1}^{2}+n_{2}^{2}}-\frac{1}{e^{R}}\left(\xi_{1} n_{1}+\xi_{2} n_{2}\right)\right],
\end{aligned}
$$

$$
T_{21}\left(M_{\circ}, Q\right)=\frac{D}{e^{R}} \frac{\sqrt{\lambda_{1}} A_{2}}{\lambda_{1} n_{1}^{2}+n_{2}^{2}} \times
$$$$
\left[\left(\lambda_{1}-1\right) n_{1} n_{2}\left(1+\frac{2}{e^{R}} \frac{\lambda_{1} n_{1} \xi_{1}+n_{2} \xi_{2}}{\lambda_{1} n_{1}^{2}+n_{2}^{2}}\right)-\frac{1}{e^{R}}\left(\lambda_{1} \xi_{1} n_{2}-\xi_{2} n_{1}\right)\right]-
$$$$
\frac{D}{e^{R}} \frac{\sqrt{\lambda_{2}} A_{1}}{\lambda_{2} n_{1}^{2}+n_{2}^{2}} \times
$$$$
\left[\left(\lambda_{2}-1\right) n_{1} n_{2}\left(1+\frac{2}{e R} \frac{\lambda_{2} n_{1} \xi_{1}+n_{2} \xi_{2}}{\lambda_{2} n_{1}^{2}+n_{2}^{2}}\right)-\frac{1}{e^{R}}\left(\lambda_{2} \xi_{1} n_{2}-\xi_{2} n_{1}\right)\right],
$$

$$
\begin{aligned}
& T_{12}\left(M_{\circ}, Q\right)=\frac{D}{e} \frac{A_{1} \sqrt{\lambda_{1}}}{\lambda_{1} n_{1}^{2}+n_{2}^{2}} \times \\
& {\left[\left(1-\frac{1}{\lambda_{1}}\right) n_{1} n_{2}\left(1+\frac{2}{{ }^{2} R} \frac{\lambda_{1} n_{1} \xi_{1}+n_{2} \xi_{2}}{\lambda_{1} n_{1}^{2}+n_{2}^{2}}\right)-\frac{1}{e^{R}}\left(\xi_{1} n_{2}-\frac{1}{\lambda_{1}} \xi_{2} n_{1}\right)\right] \times} \\
& \quad-D \frac{A_{2} \sqrt{\lambda_{2}}}{\lambda_{2} n_{1}^{2}+n_{2}^{2}} \times \\
& {\left[\left(1-\frac{1}{\lambda_{2}}\right) n_{1} n_{2}\left(1+\frac{2}{{ }^{2} R} \frac{\lambda_{1} n_{1} \xi_{1}+n_{2} \xi_{2}}{\lambda_{1} n_{1}^{2}+n_{2}^{2}}\right)-\frac{1}{{ }^{e} R}\left(\xi_{1} n_{2}-\frac{1}{\lambda_{2}} \xi_{2} n_{1}\right)\right],}
\end{aligned}
$$




$$
\begin{aligned}
& T_{22}\left(M_{\circ}, Q\right)= \\
& \quad \frac{D}{e R} \frac{\sqrt{\lambda_{1}} A_{1}}{\lambda_{1} n_{1}^{2}+n_{2}^{2}}\left[1+\frac{2}{e R} \frac{\lambda_{1} n_{1} \xi_{1}+n_{2} \xi_{2}}{\lambda_{1} n_{1}^{2}+n_{2}^{2}}-\frac{1}{e R}\left(\xi_{1} n_{1}-\xi_{2} n_{2}\right)\right]- \\
& -\frac{D}{e R} \frac{\sqrt{\lambda_{2}} A_{2}}{\lambda_{2} n_{1}^{2}+n_{2}^{2}}\left[1+\frac{2}{e^{R}} \frac{\lambda_{2} n_{1} \xi_{1}+n_{2} \xi_{2}}{\lambda_{2} n_{1}^{2}+n_{2}^{2}}-\frac{1}{e R}\left(\xi_{1} n_{1}-\xi_{2} n_{2}\right)\right] .
\end{aligned}
$$

Knowledge of the series of $U_{\lambda \kappa}$ and $T_{\lambda \kappa}$ allows us to calculate the integrals $\stackrel{(3)}{I}_{\kappa}$ and $\stackrel{(4)}{I}_{\kappa}$. The following observations are used in the calculations:

1 The outward unit normal on $\mathcal{L}_{R}$ is given by equation

$$
n_{a}=(\sin \varphi, \cos \varphi)
$$

where $\varphi$ is the polar angle.

2 The arc element on $\mathcal{L}_{R}$ admits the form

$$
\mathrm{d} s_{o}={ }_{e} R \mathrm{~d} \varphi .
$$

3 As ${ }_{e} R \rightarrow \infty$ the coefficient(s) of ${ }_{e} R$ is (are) always an integral (integrals) of zero value. The integrals we have used are all listed in the Appendix.

4 The structure of the coefficients of ${ }_{e} R$ to the power zero is similar; but these terms involve $\xi_{\alpha}$ and the trigonometric integrals that constitute these coefficients are not necessarily equal to zero.

After performing the integrations for $I_{\kappa}$ we have

$$
\begin{aligned}
& \stackrel{(3)}{I_{\kappa}}=\lim _{e R \rightarrow \infty} t_{\lambda \rho}(\infty) \oint_{\mathcal{L}_{R}} n_{\rho}\left(M_{\circ}\right) U_{\lambda \kappa}\left(M_{\circ}, Q\right) \mathrm{d} s_{M_{\odot}}= \\
& =t_{11}(\infty)\left(\xi_{1} I_{\kappa 1}^{(31)}+\xi_{2} \stackrel{(31)}{I}_{\kappa 2}\right)+t_{12}(\infty)\left(\xi_{1} I_{\kappa 1}^{(32)}+\xi_{2} \stackrel{(32)}{I}_{\kappa 2}\right)+ \\
& +t_{21}(\infty)\left(\xi_{1} \stackrel{(33)}{I}_{\kappa 1}+\xi_{2} \stackrel{(33)}{I}_{\kappa 2}\right)+t_{22}(\infty)\left(\xi_{1} I_{\kappa 1}^{(34)}+\xi_{2} \stackrel{(34)}{I}_{\kappa 2}\right),
\end{aligned}
$$

where

$$
\begin{aligned}
& \stackrel{(31)}{I_{11}}=2 \pi D\left\{A_{1}^{2} \frac{\lambda_{2}}{1+\sqrt{\lambda_{2}}}-A_{2}^{2} \frac{\lambda_{1}}{1+\sqrt{\lambda_{1}}}\right\} \\
& \stackrel{(32)}{I_{12}}=2 \pi D\left\{A_{1}^{2} \frac{\sqrt{\lambda_{2}}}{1+\sqrt{\lambda_{2}}}-A_{2}^{2} \frac{\sqrt{\lambda_{1}}}{1+\sqrt{\lambda_{1}}}\right\} \text {, } \\
& \stackrel{(32)}{I_{11}}=0 \text {, } \\
& \stackrel{(33)}{I_{12}}=-2 \pi D A_{1} A_{2} \frac{\sqrt{\lambda_{1}}-\sqrt{\lambda_{2}}}{\left(\sqrt{\lambda_{1}}+1\right)\left(\sqrt{\lambda_{2}}+1\right)}, \\
& \stackrel{(33)}{I_{11}}=0 \text {, } \\
& \stackrel{(34)}{I_{11}}=\stackrel{(33)}{I_{12}}, \\
& \stackrel{(34)}{I} \underset{12}{ }=0 \text {, } \\
& \text { (31) (33) } \\
& \text { (32) (34) } \\
& \stackrel{(32)}{I_{21}}=\stackrel{(34)}{I_{11}} \text {, } \\
& I_{21}^{(33)}=2 \pi D\left\{A_{1}^{2} \frac{1}{\sqrt{\lambda_{1}}+1}-A_{2}^{2} \frac{1}{\sqrt{\lambda_{2}}+1}\right\}, \\
& \stackrel{(34)}{I_{22}}=2 \pi D\left\{\frac{A_{1}^{2}}{\sqrt{\lambda_{1}}} \frac{1}{\sqrt{\lambda_{1}}+1}-\frac{A_{2}^{2}}{\sqrt{\lambda_{2}}} \frac{1}{\sqrt{\lambda_{2}}+1}\right\}, \quad \stackrel{(34)}{I_{21}}=0 .
\end{aligned}
$$$$
\stackrel{(31)}{I_{12}}=0, \quad(34 \mathrm{a})
$$
$\stackrel{(31)}{I} 21=0, \quad(34 \mathrm{e})$

(4)

In the same way we obtain for $I_{\kappa}$ that

$$
\begin{aligned}
& \stackrel{(4)}{I_{\kappa}}=\lim _{e R \rightarrow \infty} e_{\lambda \beta}(\infty)_{e} R \oint_{\mathcal{L}_{R}} n_{\beta}(\stackrel{o}{M}) T_{\lambda \kappa}\left(M_{\circ}, Q\right) \mathrm{d} s_{M_{\circ}}= \\
& =e_{11}(\infty)\left(\xi_{1} I_{\kappa 1}^{(41)}+\xi_{2} \stackrel{(41)}{I}_{\kappa 2}\right)+e_{12}(\infty)\left(\xi_{1} I_{\kappa 1}^{(42)}+\xi_{2} I_{\kappa 2}^{(42)}\right)+ \\
& \left.+e_{21}(\infty)\left(\xi_{1} I_{\kappa 1}^{(43)}+\xi_{2} \stackrel{(43)}{I}\right)_{\kappa 2}\right)+e_{22}(\infty)\left(\xi_{1} I_{\kappa 1}^{(44)}+\xi_{2} I_{\kappa 2}^{(44)}\right),
\end{aligned}
$$

where

$$
\begin{aligned}
& \stackrel{(41)}{I_{11}}=2 \pi D\left\{A_{1} \frac{\sqrt{\lambda_{2}}}{\sqrt{\lambda_{2}}+1}-A_{2} \frac{\sqrt{\lambda_{1}}}{\sqrt{\lambda_{1}}+1}\right\}, \quad \stackrel{(41)}{I_{12}}=0, \quad \text { (36a) } \\
& \stackrel{(42)}{I_{12}}=2 \pi D\left\{A_{1} \frac{\sqrt{\lambda_{2}}-1}{\lambda_{2}-1}-A_{2} \frac{\sqrt{\lambda_{1}}-1}{\lambda_{1}-1}\right\}, \quad \stackrel{(42)}{I_{11}}=0, \quad \text { (36b) } \\
& \stackrel{(43)}{I_{12}}=2 \pi D\left\{A_{2} \frac{\lambda_{1}-\sqrt{\lambda_{1}}}{\left(\lambda_{1}-1\right)}-A_{1} \frac{\lambda_{2}-\sqrt{\lambda_{2}}}{\left(\lambda_{2}-1\right)}\right\}, \quad \begin{array}{l}
I_{11} \\
I_{11}
\end{array}=0, \quad \text { (36c) } \\
& \stackrel{(44)}{I_{11}}=-2 \pi D\left\{A_{2} \frac{\lambda_{1}\left(\sqrt{\lambda_{1}}-1\right)}{\left(\lambda_{1}-1\right)}-A_{1} \frac{\lambda_{2}\left(\sqrt{\lambda_{2}}-1\right)}{\left(\lambda_{2}-1\right)}\right\}, \quad \stackrel{(44)}{I_{12}}=0, \quad \text { (36d) } \\
& \left.\stackrel{(41)}{I_{22}}=2 \pi D\left\{A_{1} \frac{\lambda_{1}-\sqrt{\lambda_{1}}}{\lambda_{1}\left(\lambda_{1}-1\right)}-A_{2} \frac{\lambda_{2}-\sqrt{\lambda_{2}}}{\lambda_{2}\left(\lambda_{2}-1\right)}\right\}, \quad \stackrel{(41)}{I_{21}}=0, \quad \text { (36e }\right) \\
& \stackrel{(42)}{I_{21}}=-2 \pi D\left\{A_{1} \frac{\sqrt{\lambda_{1}}-1}{\lambda_{1}-1}-A_{2} \frac{\sqrt{\lambda_{2}}-1}{\lambda_{2}-1}\right\}, \quad \stackrel{(42)}{I_{22}}=0, \quad \text { (36f) } \\
& \stackrel{(43)}{I_{21}}=2 \pi D\left\{A_{1} \frac{\sqrt{\lambda_{1}}}{\sqrt{\lambda_{1}}+1}-A_{2} \frac{\sqrt{\lambda_{2}}}{\sqrt{\lambda_{2}}+1}\right\}, \quad \stackrel{(43)}{I_{22}}=0, \quad(36 \mathrm{~g})
\end{aligned}
$$

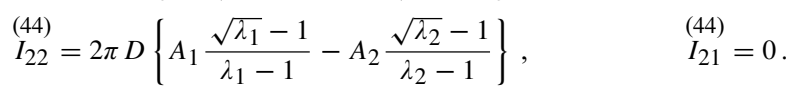

Substituting the integrals with zero value from Eqs. (34a),..., 34h and 36a, ,..., 36h into Eqs. (33) and (35) then utilizing the Hooke law (17), (3) we obtain

$$
\begin{aligned}
& \stackrel{(3)}{I_{1}}+\stackrel{(4)}{I_{1}}=\frac{s_{22} e_{11}(\infty)-s_{12} e_{22}(\infty)}{s_{11} s_{22}-s_{12}^{2}} \xi_{1} \stackrel{(31)}{I}_{11}+ \\
& \frac{2}{s_{66}} e_{12}(\infty) \xi_{2}\left(\stackrel{(32)}{I}_{12}+\stackrel{(33)}{I_{12}}\right)+ \\
& \frac{s_{11} e_{22}(\infty)-s_{21} e_{11}(\infty)}{s_{11} s_{22}-s_{12}^{2}} \xi_{1} \stackrel{(34)}{I_{11}}-e_{11}(\infty)\left(\xi_{1} \stackrel{(41)}{I}_{11}+\xi_{2} \stackrel{(41)}{I}_{12}\right)- \\
& e_{12}(\infty)\left[\xi_{1}\left(\stackrel{(42)}{I}_{11}+\stackrel{(43)}{I}_{11}\right)+\xi_{2}\left(\stackrel{(42)}{I}_{12}+\stackrel{(43)}{I_{12}}\right)\right]- \\
& e_{22}(\infty)\left(\xi_{1} \stackrel{(44)}{I_{11}}+\xi_{2} \stackrel{(44)}{I_{12}}\right)
\end{aligned}
$$

and

$$
\begin{aligned}
& \stackrel{(3)}{I_{2}}+\stackrel{(4)}{I_{2}}=\frac{s_{22} e_{11}(\infty)-s_{12} e_{22}(\infty)}{s_{11} s_{22}-s_{12}^{2}} \xi_{2} \stackrel{(31)}{I} \underset{22}{ }+ \\
& \frac{2}{s_{66}} e_{12}(\infty) \xi_{1}\left(\stackrel{(32)}{I} 21+\stackrel{(33)}{I_{21}}\right)+ \\
& \frac{s_{11} e_{22}(\infty)-s_{21} e_{11}(\infty)}{s_{11} s_{22}-s_{12}^{2}} \xi_{2} \stackrel{(34)}{I_{22}}-e_{11}(\infty)\left(\xi_{1} \stackrel{(41)}{I}_{21}+\xi_{2} \stackrel{(41)}{I_{22}}\right)- \\
& e_{12}(\infty)\left[\xi_{1}\left(\stackrel{(42)}{I}_{21}+\stackrel{(43)}{I_{21}}\right)+\xi_{2}\left(\stackrel{(42)}{I}_{22}+\stackrel{(43)}{I}_{22}\right)\right]- \\
& e_{22}(\infty)\left(\xi_{1} \stackrel{(44)}{I_{21}}+\stackrel{\xi}{\xi}_{2} \stackrel{(44)}{I_{22}}\right) \text {. }
\end{aligned}
$$

Using Eqs. (7) to (12), one can prove that the following equations hold (the formal calculations are presented in Appendix B.):

$$
\begin{array}{r}
-\frac{s_{22}}{s_{12}^{2}-s_{11} s_{22}} \stackrel{(31)}{I_{11}}+\frac{s_{21}(\infty)}{s_{12}^{2}-s_{11} s_{22}} \stackrel{(34)}{I_{11}}-\stackrel{(41)}{I_{11}}=1, \\
\frac{2}{s_{66}}\left(\stackrel{(32)}{I_{12}}+\stackrel{(33)}{I_{12}}\right)-\stackrel{(42)}{I_{12}}-\stackrel{(43)}{I_{12}}=1, \\
\frac{s_{12}}{s_{12}^{2}-s_{11} s_{22}} \stackrel{(31)}{I}_{11}-\frac{s_{11}}{s_{12}^{2}-s_{11} s_{22}} \stackrel{(34)}{I_{11}}-\stackrel{(44)}{I_{11}}=0
\end{array}
$$


and

$$
\begin{array}{r}
-\frac{s_{22}}{s_{12}^{2}-s_{11} s_{22}} \stackrel{(31)}{I}_{22}+\frac{s_{21}}{s_{12}^{2}-s_{11} s_{22}} \stackrel{(34)}{I_{22}}-\stackrel{(41)}{I_{22}}=0, \\
\frac{2}{s_{66}}\left(\stackrel{(32)}{I_{21}}+\stackrel{(33)}{I_{21}}\right)-\stackrel{(42)}{I}_{21}-\stackrel{(43)}{I_{21}}=1, \\
\frac{s_{12} \quad \stackrel{(31)}{I_{22}}-\frac{s_{11}}{s_{12}^{2}-s_{11} s_{22}} \stackrel{(34)}{I}_{22}-\stackrel{(44)}{I}_{22}=1 .}{s_{11} s_{22}}=1 .
\end{array}
$$

If we substitute Eqs. (38a,b) into equtions $(37 \mathrm{a}, \mathrm{b})$ we find that

$$
\stackrel{(3)}{I_{\kappa}}+\stackrel{(4)}{I}_{\kappa}=e_{\kappa \beta}(\infty) \xi_{\beta} \text {. }
$$

Neglecting the rigid body motion, i.e., setting $\stackrel{(1)}{I}_{\kappa}+\stackrel{(2)}{I_{\kappa}}$ to zero and utilizing (39) we obtain

$$
I_{\kappa}=I_{\kappa}=\stackrel{(1)}{I_{\kappa}}+\stackrel{(2)}{I_{\kappa}}+\stackrel{(3)}{I^{\prime}}+\stackrel{(4)}{I_{\kappa}}=e_{\kappa \beta}(\infty) \xi_{\beta} .
$$

Then the first and modified Somigliana formula immediately follow from Eqs. (19) and (20):

$$
\begin{array}{r}
u_{\kappa}(Q)=e_{\kappa \beta}(\infty) \xi_{\beta}(Q)+\oint_{\mathcal{L}_{o}}\left[t_{\lambda}\left(M_{\circ}\right) U_{\lambda \kappa}\left(M_{\circ}, Q\right)-\right. \\
\left.u_{\lambda}\left(M_{\circ}\right) T_{\lambda \kappa}\left(M_{\circ}, Q\right)\right] \mathrm{d} s_{M_{\circ}} \quad Q \in A_{e}
\end{array}
$$

If $Q=Q_{\circ}$ is on $\mathcal{L}_{o}$ nothing changes concerning the limit of the integral taken on $\mathcal{L}_{R}$. Consequently

$$
\begin{aligned}
& C_{\kappa \rho} u_{\rho}\left(Q_{\circ}\right)=e_{\kappa \beta}(\infty) \xi_{\beta}\left(Q_{\circ}\right)+ \\
& \oint_{\mathcal{L}_{o}}\left[t_{\lambda}\left(M_{\circ}\right) U_{\lambda \kappa}\left(M_{\circ}, Q_{\circ}\right)-u_{\lambda}\left(M_{\circ}\right) T_{\lambda \kappa}\left(M_{\circ}, Q_{\circ}\right)\right] \mathrm{d} s_{M_{\circ}} \\
& Q=Q_{\circ} \in \mathcal{L}_{o}
\end{aligned}
$$

where $C_{\kappa \rho}=\delta_{\kappa \rho} / 2$ if the contour is smooth at $Q_{\circ}$. This integral equation is that of the direct method (or the second Somigliana formula for exterior regions).

If $Q$ is inside the contour $\mathcal{L}_{o}$ - this region is referred to as $A_{i}$ - then it is easy to show that

$$
\begin{aligned}
& 0=e_{\kappa \beta}(\infty) \xi_{\beta}(Q)+ \\
& \oint_{\mathcal{L}_{o}}\left[t_{\lambda}\left(M_{\circ}\right) U_{\lambda \kappa}\left(M_{\circ}, Q\right)-u_{\lambda}\left(M_{\circ}\right) T_{\lambda \kappa}\left(M_{\circ}, Q\right)\right] \mathrm{d} s_{M_{\circ}} \\
& Q=Q_{\circ} \in A_{i}
\end{aligned}
$$

which is the third Somigliana formula for exterior regions.

Using the formulae set up for the strains in Appendix $\mathrm{C}$ and the Hooke law (17) we can calculate the stresses:

$$
\begin{array}{r}
t_{\alpha \beta}(Q)=t_{\alpha \beta}(\infty)+\oint_{\mathcal{L}_{o}} t_{\lambda}\left(M_{\circ}\right) \hat{\mathcal{D}}_{\lambda \alpha \beta}\left(M_{\circ}, Q\right) \mathrm{d} s_{M_{\circ}}- \\
\oint_{\mathcal{L}_{o}} u_{\lambda}\left(M_{\circ}\right) \mathcal{S}_{\lambda \alpha \beta}\left(M_{\circ}, Q\right) \mathrm{d} s_{M_{\circ}} Q \in A_{e}
\end{array}
$$

where

$$
\begin{array}{ll}
\hat{\mathcal{D}}_{\lambda 11}=c_{11} \mathcal{D}_{\lambda 11}+c_{12} \mathcal{D}_{\lambda 22}, & \hat{\mathcal{S}}_{\lambda 11}=c_{11} \mathcal{S}_{\lambda 11}+c_{12} \mathcal{S}_{\lambda 22}, \\
\hat{\mathcal{D}}_{\lambda 11}=c_{12} \mathcal{D}_{\lambda 11}+c_{22} \mathcal{D}_{\lambda 22}, & \hat{\mathcal{S}}_{\lambda 11}=c_{12} \mathcal{S}_{\lambda 11}+c_{22} \mathcal{S}_{\lambda 22}, \\
\hat{\mathcal{D}}_{\lambda 12}=2 c_{66} \mathcal{D}_{\lambda 12}=\hat{\mathcal{D}}_{\lambda 21}, & \hat{\mathcal{S}}_{\lambda 12}=2 c_{66} \mathcal{S}_{\lambda 12}=\hat{\mathcal{S}}_{\lambda 21} .
\end{array}
$$

\section{Behaviour at infinity}

Our goal in this section is to compute the limit of representation (40) as $Q \rightarrow \infty$. This will lead to a characterization of the asymptotic behaviour of $u_{\kappa}(Q)$. If this behaviour is the same what we have assumed, i.e., if the limit coincides with (16) provided that in the latter the rigid body motion is neglected, then we confirm that the results of the previous section are correct. It is clear from representation (40) that it is sufficient to show that the following relations hold

$$
\begin{aligned}
\lim _{Q\left(\xi_{1}, \xi_{2}\right) \rightarrow \infty} & \oint_{\mathcal{L}_{o}} t_{\lambda}\left(M_{\circ}\right) U_{\lambda \kappa}\left(M_{\circ}, Q\right) \mathrm{d} s_{M_{\circ}}=0, \\
& \lim _{Q\left(\xi_{1}, \xi_{2}\right) \rightarrow \infty} \oint_{\mathcal{L}_{o}} u_{\lambda}\left(M_{\circ}\right) T_{\lambda \kappa}\left(M_{\circ}, Q\right) \mathrm{d} s_{M_{\circ}}=0 .
\end{aligned}
$$

In order to find the limit of the above integrals we have to set up asymptotic relations for the fundamental solutions $U_{\lambda \kappa}\left(M_{\circ}, Q\right)$ and $T_{\lambda_{\kappa}}\left(M_{\circ}, Q\right)$ if $Q \rightarrow \infty$.

Using the notations introduced in Fig. 2 as well as Eqs. (6) and (10) we have

$$
\begin{gathered}
r_{\alpha}(\stackrel{o}{M}, Q)=x_{\alpha}(\stackrel{o}{M})-\xi_{\alpha}(Q)=x_{\alpha}-\xi_{\alpha}= \\
-\hat{R}\left(\hat{n}_{\alpha}-\frac{x_{\alpha}}{\hat{R}}\right) \approx-\hat{R} \hat{n}_{\alpha}\left|\hat{n}_{\alpha}\right|=1, \\
\rho_{\alpha}=\sqrt{\lambda_{\alpha} r_{1}^{2}+r_{2}^{2}} \approx \hat{R} \sqrt{\lambda_{\alpha} \hat{n}_{1}^{2}+\hat{n}_{2}^{2}}, \\
\ln \rho_{\alpha} \approx \ln \hat{R}+\frac{1}{2} \ln \left(\lambda_{\alpha} \hat{n}_{1}^{2}+\hat{n}_{2}^{2}\right) .
\end{gathered}
$$

Substituting equations $46 \mathrm{~b}, \mathrm{~b}, \mathrm{c})$ into 13 and then performing

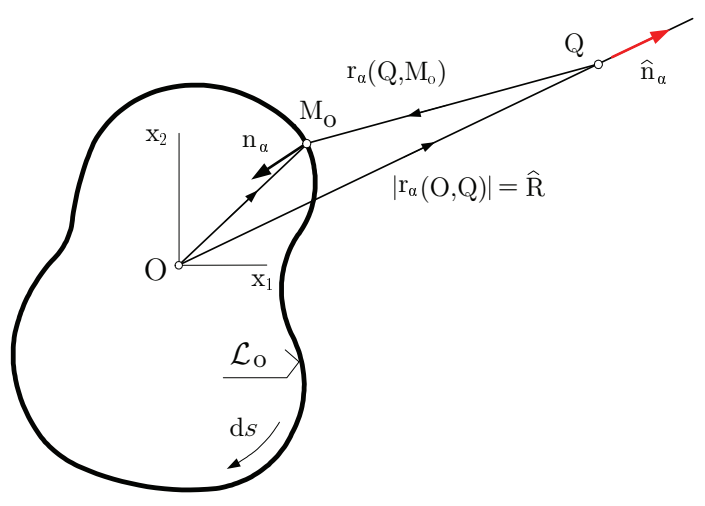

Fig. 2.

some manipulations, we obtain the following asymptotic relations for the fundamental solution of order one:

$$
\begin{array}{r}
U_{11}\left(M_{\circ}, Q\right) \approx D\left[\sqrt{\lambda_{1}} A_{2}^{2}-\sqrt{\lambda_{2}} A_{1}^{2}\right] \ln \hat{R}, \\
U_{12}\left(M_{\circ}, Q\right)=U_{21}\left(M_{\circ}, Q\right) \approx \\
D A_{1} A_{2} \arctan \frac{\left(\sqrt{\lambda_{1}}-\sqrt{\lambda_{2}}\right) \hat{n}_{1} \hat{n}_{2}}{\sqrt{\lambda_{1}} \sqrt{\lambda_{2}} \hat{n}_{1}^{2}+\hat{n}_{2}^{2}}, \\
U_{22}\left(M_{\circ}, Q\right) \approx-D\left[\frac{A_{1}^{2}}{\sqrt{\lambda_{1}}}-\frac{A_{2}^{2}}{\sqrt{\lambda_{2}}}\right] \ln \hat{R} .
\end{array}
$$

It is obvious that asymptotically $U_{\lambda \kappa}\left(M_{\circ}, Q\right) \approx U_{\lambda \kappa}(Q)$, i.e., the kernel in integral $(45)_{1}$ is independent of $M_{\circ}$. 
Consequently

$$
\begin{aligned}
\lim _{Q\left(\xi_{1}, \xi_{2}\right) \rightarrow \infty} & \oint_{\mathcal{L}_{o}} t_{\lambda}\left(M_{\circ}\right) U_{\lambda \kappa}\left(M_{\circ}, Q\right) \mathrm{d} s_{M_{\circ}}= \\
& =\lim _{Q\left(\xi_{1}, \xi_{2}\right) \rightarrow \infty} U_{\lambda \kappa}(Q) \underbrace{\oint_{\mathcal{L}_{o}} t_{\lambda}\left(M_{\circ}\right) \mathrm{d} s_{M_{\circ}}}_{\text {resultant }}=0 .
\end{aligned}
$$

By repeating the line of thought leading to the asymptotic relations $47 \mathrm{a}, \mathrm{b}, \mathrm{c})$, for the fundamental solutions of order two we obtain

$T_{11}\left(M_{\circ}, Q\right) \approx$

$-\frac{D}{\hat{R}}\left(\frac{\sqrt{\lambda_{2}} A_{1}}{\lambda_{2} \hat{n}_{1}^{2}+\hat{n}_{2}^{2}}-\frac{\sqrt{\lambda_{1}} A_{2}}{\lambda_{1} \hat{n}_{1}^{2}+\hat{n}_{2}^{2}}\right)=\frac{\tilde{T}_{11}(Q)}{\hat{R}}$,

$T_{12}\left(M_{\circ}, Q\right) \approx$

$-\frac{D}{\hat{R}}\left[\frac{1-\lambda_{2}}{\sqrt{\lambda_{2}}} \frac{A_{2}}{\lambda_{2} \hat{n}_{1}^{2}+\hat{n}_{2}^{2}}-\frac{1-\lambda_{1}}{\sqrt{\lambda_{1}}} \frac{A_{1}}{\lambda_{1} \hat{n}_{1}^{2}+\hat{n}_{2}^{2}}\right] \hat{n}_{1} \hat{n}_{2}=\frac{\tilde{T}_{12}(Q)}{\hat{R}}$,

$T_{21}\left(M_{\circ}, Q\right) \approx$

$-\frac{D}{\hat{R}}\left[\frac{\sqrt{\lambda_{1}}\left(\lambda_{1}-1\right) A_{2}}{\lambda_{1} \hat{n}_{1}^{2}+\hat{n}_{2}^{2}}-\frac{\sqrt{\lambda_{2}}\left(\lambda_{2}-1\right) A_{1}}{\lambda_{2} \hat{n}_{1}^{2}+\hat{n}_{2}^{2}}\right] \hat{n}_{1} \hat{n}_{2}=\frac{\tilde{T}_{21}(Q)}{\hat{R}}$,

$T_{22}\left(M_{\circ}, Q\right) \approx$

$-\frac{D}{\hat{R}}\left(\frac{\sqrt{\lambda_{2}} A_{1}}{\lambda_{1} \hat{n}_{1}^{2}+\hat{n}_{2}^{2}}-\frac{\sqrt{\lambda_{1}} A_{2}}{\lambda_{2} \hat{n}_{1}^{2}+\hat{n}_{2}^{2}}\right)=\frac{\tilde{T}_{22}(Q)}{\hat{R}}$,

where $\tilde{T}_{\lambda \kappa}=\tilde{T}_{\lambda \kappa}(Q)$ is defined by the above equations. Since for large $\hat{R}$ the kernel $T_{\lambda \kappa}\left(M_{\circ}, Q\right)$ is independent of $M_{\circ}$ and tends to zero, it follows that

$$
\begin{aligned}
\lim _{Q\left(\xi_{1}, \xi_{2}\right) \rightarrow \infty} & \oint_{\mathcal{L}_{o}} u_{\lambda}\left(M_{\circ}\right) T_{\lambda \kappa}\left(M_{\circ}, Q\right) \mathrm{d} s_{M_{\circ}}= \\
& =\lim _{Q\left(\xi_{1}, \xi_{2}\right) \rightarrow \infty} \frac{\tilde{T}_{\lambda \kappa}(Q)}{\hat{R}} \oint_{\mathcal{L}_{o}} u_{\lambda}\left(M_{\circ}\right) \mathrm{d} s_{M_{\circ}}=0 .
\end{aligned}
$$

This verifies that the asymptotic behaviour of the displacement representation (40) is as expected.

\section{Concluding remarks}

For the sake of completeness, note that Constanda [3] gives an asymptotic expansion for the displacements at infinity which ensures the validity of the Betti formula for exterior regions and isotropic bodies. Under this condition the total strain energy stored in the region is bounded. In addition uniqueness and existence proofs are easy to give.

We have modified the Somigliana formulas for exterior regions of orthotropic bodies by assuming that the strains are constants and accordingly the displacements are linear at infinity. Under this condition the strain energy density is bounded (although the strain energy is not), and there is no need to replace the exterior region by a finite one if a constant stress condition is prescribed at infinity. This can be an advantage if one considers an infinite plane with holes or cracks subjected to constant stresses at infinity, and an attempt is made to determine the stresses in finite. Existing codes can be modified easily to perform computations.

\section{References}

1 Banarjee PK, Butterfield R, Boundary Element Methods in Engineering Science, Mir, Moscow, 1984.

2 Banarjee PK, The Bondary Element Methods in Engineering, McGrawHill, New York, 1994.

3 Constanda C, The Boundary Iintegral Equation Method in Plane Elasticity, Proceedings of the American Mathematical Society 123 (1995), no. 11, 3385-3396.

4 , Iintegral Equations of the First Kind in Plane Elasticity, Quarterly of Applied Mathematics LIII (1995), no. 4, 783-793.

5 Gradstein IS, Ryzhik IM, Table of Integrals, Series and Products, Academic, New York, 1980.

6 , Table of Integrals, Series and Products, Moscow, Nauka Pub. (in Russian), 1963.

7 Huang L, Sun X, Liu Y, Cen Z, Parameter identification for twodimensional orthotropic material bodies by the boundary element method, Engineering Analysis with Boundary Elements 28 (2004), no. 2, 109-121.

8 Vable M, Sikarskie DL, Stress analysis in plane orthotropic material by the boundary element method, Int. J. Solids Structures 24 (1988), no. 1, 1-11.

9 Rizzo RJ, Shippy DJ, A method for stress determination in plane anisotropic elastic bodies, J. Composite Materials 4 (1970), 36-61.

10 Schiavone $\mathbf{P}$, Chong-Quing $\mathbf{R u}$, On the exterior mixed problem in plane elasticity, Mathematics and Mechanics of Solids 1 (1996), 335-342.

11 Szeidl Gy, Boundary integral equations for plane problems - remark to the formulation for exterior regions, Publications of the University of Miskolc, Series D, Natural Sciences, Mathematics 40 (1999), no. 1, 79-88.

12 _ Boundary integral equations for plane problems in terms of stress functions of order one, Journal of Computational and Applied Mechanics 2 (2001), no. 2, 237-261. 


\section{A Trigonometric integrals}

When determining the integrals $\stackrel{(3)}{I}_{\kappa}+\stackrel{(4)}{I}_{\kappa}$ we have used the following trigonometric integrals:

$$
\begin{aligned}
& \frac{1}{{ }_{e} R} \oint_{\mathcal{L}_{R}} n_{1} \ln _{e} R \mathrm{~d} s=\ln { }_{e} R \int_{0}^{2 \pi} \cos \psi \mathrm{d} \psi=0, \quad \frac{1}{{ }_{e} R} \oint_{\mathcal{L}_{R}} n_{2} \ln { }_{e} R \mathrm{~d} s=\ln _{e} R \int_{0}^{2 \pi} \sin \psi \mathrm{d} \psi=0, \\
& \frac{1}{{ }_{e} R} \oint_{\mathcal{L}_{R}} n_{1} \ln \left[\lambda_{\alpha} n_{1}^{2}+n_{2}^{2}\right] \mathrm{d} s=\int_{0}^{2 \pi}(\cos \psi) \ln \left[\lambda_{\alpha} \cos ^{2} \psi+\sin ^{2} \psi\right] \mathrm{d} \psi=0, \\
& \frac{1}{{ }_{e} R} \oint_{\mathcal{L}_{R}} n_{2} \ln \left[\lambda_{\alpha} n_{1}^{2}+n_{2}^{2}\right] \mathrm{d} s=\int_{0}^{2 \pi}(\sin \psi) \ln \left[\lambda_{\alpha} \cos ^{2} \psi+\sin ^{2} \psi\right] \mathrm{d} \psi=0, \\
& \frac{1}{{ }_{e} R} \oint_{\mathcal{L}_{R}} \frac{n_{1}^{2}}{\lambda_{\alpha} n_{1}^{2}+n_{2}^{2}} \mathrm{~d} s=\int_{0}^{2 \pi} \frac{\cos ^{2} \psi}{\lambda_{\alpha} \cos ^{2} \psi+\sin ^{2} \psi} \mathrm{d} \psi=\frac{2 \pi}{\lambda_{\alpha}+\sqrt{\lambda_{\alpha}}}, \\
& \frac{1}{{ }_{e} R} \oint_{\mathcal{L}_{R}} \frac{n_{1} n_{2}}{\lambda_{\alpha} n_{1}^{2}+n_{2}^{2}} \mathrm{~d} s=\int_{0}^{2 \pi} \frac{\cos \psi \sin \psi}{\lambda_{\alpha} \cos ^{2} \psi+\sin ^{2} \psi} \mathrm{d} \psi=0 \\
& \frac{1}{{ }_{e} R} \oint_{\mathcal{L}_{R}} \frac{n_{2}^{2}}{\lambda_{\alpha} n_{1}^{2}+n_{2}^{2}} \mathrm{~d} s=\int_{0}^{2 \pi} \frac{\sin ^{2} \psi}{\lambda_{\alpha} \cos ^{2} \psi+\sin ^{2} \psi} \mathrm{d} \psi=\frac{2 \pi}{\sqrt{\lambda_{a}}+1}, \\
& \frac{1}{{ }_{e} R} \oint_{\mathcal{L}_{R}} \frac{n_{1}^{3} n_{2}}{\left(\lambda_{1} n_{1}^{2}+n_{2}^{2}\right)\left(\lambda_{1} n_{1}^{2}+n_{2}^{2}\right)} \mathrm{d} s=\int_{0}^{2 \pi} \frac{\cos ^{3} \psi \sin \psi}{\left(\lambda_{1} \cos ^{2} \psi+\sin ^{2} \psi\right)\left(\lambda_{2} \cos ^{2} \psi+\sin ^{2} \psi\right)} \mathrm{d} \psi=0, \\
& \frac{1}{{ }_{e} R} \oint_{\mathcal{L}_{R}} \frac{n_{1} n_{2}^{3}}{\left(\lambda_{1} n_{1}^{2}+n_{2}^{2}\right)\left(\lambda_{1} n_{1}^{2}+n_{2}^{2}\right)} \mathrm{d} s=\int_{0}^{2 \pi} \frac{\cos \psi \sin ^{3} \psi}{\left(\lambda_{1} \cos ^{2} \psi+\sin ^{2} \psi\right)\left(\lambda_{2} \cos ^{2} \psi+\sin ^{2} \psi\right)} \mathrm{d} \psi=0, \\
& \frac{1}{{ }_{e} R} \oint_{\mathcal{L}_{R}} \frac{n_{1}^{4}}{\left(\lambda_{1} n_{1}^{2}+n_{2}^{2}\right)\left(\lambda_{2} n_{1}^{2}+n_{2}^{2}\right)} \mathrm{d} s= \\
& -\int_{0}^{2 \pi} \frac{\cos ^{4} \psi}{\left(\lambda_{1} \cos ^{2} \psi+\sin ^{2} \psi\right)\left(\lambda_{2} \cos ^{2} \psi+\sin ^{2} \psi\right)} \mathrm{d} \psi=2 \pi \frac{\sqrt{\lambda_{1}}+\sqrt{\lambda_{2}}+1}{\sqrt{\lambda_{1} \lambda_{2}}\left(\sqrt{\lambda_{1}}+\sqrt{\lambda_{2}}\right)\left(\sqrt{\lambda_{1}}+1\right)\left(\sqrt{\lambda_{2}}+1\right)}, \\
& \frac{1}{{ }_{e} R} \oint_{\mathcal{L}_{R}} \frac{n_{1}^{2} n_{2}^{2}}{\left(\lambda_{1} n_{1}^{2}+n_{2}^{2}\right)\left(\lambda_{2} n_{1}^{2}+n_{2}^{2}\right)} \mathrm{d} s= \\
& \int_{0}^{2 \pi} \frac{\cos ^{2} \psi \sin ^{2} \psi}{\left(\lambda_{1} \cos ^{2} \psi+\sin ^{2} \psi\right)\left(\lambda_{2} \cos ^{2} \psi+\sin ^{2} \psi\right)} \mathrm{d} \psi=2 \pi \frac{1}{\left(\sqrt{\lambda_{1}}+\sqrt{\lambda_{2}}\right)\left(\sqrt{\lambda_{1}}+1\right)\left(\sqrt{\lambda_{2}}+1\right)}, \\
& \frac{1}{{ }_{e} R} \oint_{\mathcal{L}_{R}} \frac{n_{2}^{4}}{n_{1}^{4} \lambda_{1} \lambda_{2}+n_{2}^{4}+\left(\lambda_{1}+\lambda_{2}\right) n_{1}^{2} n_{2}^{2}} \mathrm{~d} s= \\
& \int_{0}^{2 \pi} \frac{\sin ^{4} \psi}{\left(\lambda_{1} \cos ^{2} \psi+\sin ^{2} \psi\right)\left(\lambda_{2} \cos ^{2} \psi+\sin ^{2} \psi\right)} \mathrm{d} \psi=2 \pi \frac{\sqrt{\lambda_{1}}+\sqrt{\lambda_{2}}+\sqrt{\lambda_{1}} \sqrt{\lambda_{2}}}{\left(\sqrt{\lambda_{1}}+\sqrt{\lambda_{2}}\right)\left(\sqrt{\lambda_{1}}+1\right)\left(\sqrt{\lambda_{2}}+1\right)} .
\end{aligned}
$$

The integrals detailed above can be checked either by using Maple 9.5 or the Table of Integrals [5,6] by Gradstein and Ryzhik.

\section{B Proof of equation $38 \mathrm{a}$}

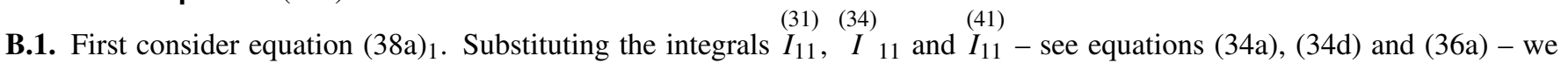
have

$$
\begin{array}{r}
-\frac{2 \pi}{s_{12}^{2}-s_{11} s_{22}}\left\{s_{22} D\left[\frac{A_{1}^{2} \lambda_{2}}{1+\sqrt{\lambda_{2}}}-\frac{A_{2}^{2} \lambda_{1}}{1+\sqrt{\lambda_{1}}}\right]+s_{21} D A_{1} A_{2} \frac{\sqrt{\lambda_{1}}-\sqrt{\lambda_{2}}}{\left(\sqrt{\lambda_{1}}+1\right)\left(\sqrt{\lambda_{2}}+1\right)}\right\}- \\
-2 \pi D A_{1} \frac{\sqrt{\lambda_{2}}}{\sqrt{\lambda_{2}}+1}+2 \pi D A_{2} \frac{\sqrt{\lambda_{1}}}{\sqrt{\lambda_{1}}+1}-1=0 .
\end{array}
$$


After multiplying throughout by the common denominator $\left(s_{12}^{2}-s_{11} s_{22}\right)\left(\lambda_{1}-1\right)\left(\lambda_{2}-1\right)$ and some further manipulations we obtain

$$
\begin{array}{r}
\left\{s_{22}\left[A_{1}^{2} \lambda_{2}\left(\sqrt{\lambda_{1}}+1\right)-A_{2}^{2} \lambda_{1}\left(\sqrt{\lambda_{2}}+1\right)\right]+s_{21} A_{1} A_{2}\left(\sqrt{\lambda_{1}}-\sqrt{\lambda_{2}}\right)+\left(s_{12}^{2}-s_{11} s_{22}\right)\left[A_{1} \sqrt{\lambda_{2}}\left(\sqrt{\lambda_{1}}+1\right)-\right.\right. \\
\left.\left.A_{2} \sqrt{\lambda_{1}}\left(\sqrt{\lambda_{2}}+1\right)\right]\right\}\left(\sqrt{\lambda_{1}}-1\right)\left(\sqrt{\lambda_{2}}-1\right)+\left(\lambda_{1}-\lambda_{2}\right) s_{22}\left(s_{12}^{2}-s_{11} s_{22}\right)\left(\lambda_{1}-1\right)\left(\lambda_{2}-1\right)=0 .
\end{array}
$$

If we divide throughout by $\left(\sqrt{\lambda_{1}}-1\right)\left(\sqrt{\lambda_{2}}-1\right)$ and substitute 9 then we find

$$
\begin{gathered}
-\left(s_{12}-\lambda_{1} s_{22}\right) \sqrt{\lambda_{2}}\left(\sqrt{\lambda_{1}}+1\right)\left[\left(s_{12}-\lambda_{1} s_{22}\right) s_{22} \sqrt{\lambda_{2}}+\left(s_{12}^{2}-s_{11} s_{22}\right)\right]+ \\
\left(s_{12}-\lambda_{2} s_{22}\right) \sqrt{\lambda_{1}}\left(\sqrt{\lambda_{2}}+1\right)\left[\left(s_{12}-\lambda_{2} s_{22}\right) s_{22} \sqrt{\lambda_{1}}+\left(s_{12}^{2}-s_{11} s_{22}\right)\right]- \\
\left(\lambda_{1}-\lambda_{2}\right) s_{22}\left(s_{12}^{2}-s_{11} s_{22}\right)\left(\sqrt{\lambda_{1}}+1\right)\left(\sqrt{\lambda_{2}}+1\right)-s_{21}\left(s_{12}-\lambda_{1} s_{22}\right)\left(s_{12}-\lambda_{2} s_{22}\right)\left(\sqrt{\lambda_{1}}-\sqrt{\lambda_{2}}\right)=0 .
\end{gathered}
$$

Here we can again divide throughout by $\sqrt{\lambda_{1}}-\sqrt{\lambda_{2}}$. Hence

$$
\begin{gathered}
-\lambda_{1}^{\frac{3}{2}} \lambda_{2}^{\frac{3}{2}} s_{22}^{3}+\lambda_{1} s_{11} s_{22}^{2}+\lambda_{2} s_{11} s_{22}^{2}-\lambda_{1} \lambda_{2}^{2} s_{22}^{3}-\lambda_{1}^{2} \lambda_{2} s_{22}^{3}-\lambda_{1} \lambda_{2}^{\frac{3}{2}} s_{22}^{3}-\lambda_{1}^{\frac{3}{2}} \lambda_{2} s_{22}^{3}+ \\
\sqrt{\lambda_{1}} s_{11} s_{22}^{2}+\sqrt{\lambda_{2}} s_{11} s_{22}^{2}-s_{11} s_{12} s_{22}+\sqrt{\lambda_{1}} \sqrt{\lambda_{2}} s_{11} s_{22}^{2}+2 \lambda_{1} \lambda_{2} s_{12} s_{22}^{2}-\lambda_{1} \lambda_{2} s_{21} s_{22}^{2}=0
\end{gathered}
$$

where

$$
\lambda_{1} s_{11} s_{22}^{2}+\lambda_{2} s_{11} s_{22}^{2}=s_{22}^{2} s_{11}\left(\lambda_{1}+\lambda_{2}\right)=s_{22}^{2} s_{11} \frac{2 s_{12}+s_{66}}{s_{22}}=\left(2 s_{12}+s_{66}\right) s_{22} s_{11}
$$

and

$$
-\lambda_{1} \lambda_{2}^{2} s_{22}^{3}-\lambda_{1}^{2} \lambda_{2} s_{22}^{3}=-\lambda_{2} \lambda_{1} s_{22}^{3}\left(\lambda_{1}+\lambda_{2}\right)=-\frac{s_{11}}{s_{22}} s_{22}^{3} \frac{2 s_{12}+s_{66}}{s_{22}}=-\left(2 s_{12}+s_{66}\right) s_{22} s_{11} .
$$

Consequently

$$
-\lambda_{1}^{\frac{3}{2}} \lambda_{2}^{\frac{3}{2}} s_{22}^{3}-\lambda_{1} \lambda_{2}^{\frac{3}{2}} s_{22}^{3}-\lambda_{1}^{\frac{3}{2}} \lambda_{2} s_{22}^{3}+\sqrt{\lambda_{1}} s_{11} s_{22}^{2}+\sqrt{\lambda_{2}} s_{11} s_{22}^{2}-s_{11} s_{12} s_{22}+\sqrt{\lambda_{1}} \sqrt{\lambda_{2}} s_{11} s_{22}^{2}+\lambda_{1} \lambda_{2} s_{12} s_{22}^{2}=0 .
$$

Here

$$
-\lambda_{1} \lambda_{2}^{\frac{3}{2}} s_{22}^{3}-\lambda_{1}^{\frac{3}{2}} \lambda_{2} s_{22}^{3}=-s_{22}^{3}\left(\lambda_{1} \lambda_{2}^{\frac{3}{2}}+\lambda_{1}^{\frac{3}{2}} \lambda_{2}\right)=-s_{22}^{3} \lambda_{1} \lambda_{2}\left(\sqrt{\lambda_{1}}+\sqrt{\lambda_{2}}\right)=-s_{22}^{3} \frac{s_{11}}{s_{22}}\left(\sqrt{\lambda_{1}}+\sqrt{\lambda_{2}}\right)=-s_{22}^{2} s_{11}\left(\sqrt{\lambda_{1}}+\sqrt{\lambda_{2}}\right)
$$

and

$$
\sqrt{\lambda_{1}} s_{11} s_{22}^{2}+\sqrt{\lambda_{2}} s_{11} s_{22}^{2}=s_{22}^{2} s_{11}\left(\sqrt{\lambda_{1}}+\sqrt{\lambda_{2}}\right)
$$

Therefore

$$
\begin{aligned}
- & \lambda_{1}^{\frac{3}{2}} \lambda_{2}^{\frac{3}{2}} s_{22}^{3}-s_{11} s_{12} s_{22}+\sqrt{\lambda_{1}} \sqrt{\lambda_{2}} s_{11} s_{22}^{2}+\lambda_{1} \lambda_{2} s_{12} s_{22}^{2}=-\lambda_{1}^{\frac{3}{2}} \lambda_{2}^{\frac{3}{2}} s_{22}^{3}+s_{22}^{2}\left(s_{12} \lambda_{1} \lambda_{2}-s_{22} \lambda_{1}^{\frac{3}{2}} \lambda_{2}^{\frac{3}{2}}\right)+\lambda_{1} \lambda_{2} s_{12} s_{22}^{2}= \\
& s_{22} s_{11}\left(s_{22} \sqrt{\lambda_{1}} \sqrt{\lambda_{2}}-s_{12}\right)-\lambda_{1}^{\frac{3}{2}} \lambda_{2}^{\frac{3}{2}} s_{22}^{3}+\lambda_{1} \lambda_{2} s_{12} s_{22}^{2}=s_{22} s_{11}\left(s_{22} \sqrt{\lambda_{1}} \sqrt{\lambda_{2}}-s_{12}\right)-s_{22} s_{11}\left(s_{22} \sqrt{\lambda_{1}} \sqrt{\lambda_{2}}-s_{12}\right)=0 .
\end{aligned}
$$

(32) (33) (42) (43)

B.2. Consider equation $38 \mathrm{a})_{2}$. If we substitute the integrals $I_{12}, I_{12}, I_{12}$ and $I_{12}$ - see equations (34b), 34c) 36b) and 36c and perform some manipulations we obtain

$$
\frac{4 \pi D}{s_{66}}\left[A_{1}^{2} \frac{\sqrt{\lambda_{2}}}{\sqrt{\lambda_{2}}+1}-A_{2}^{2} \frac{\sqrt{\lambda_{1}}}{\sqrt{\lambda_{1}}+1}-A_{1} A_{2} \frac{\sqrt{\lambda_{1}}-\sqrt{\lambda_{2}}}{\left(\sqrt{\lambda_{1}}+1\right)\left(\sqrt{\lambda_{2}}+1\right)}+A_{1} \frac{\sqrt{\lambda_{2}}-1}{\sqrt{\lambda_{2}}+1}-A_{2} \frac{\sqrt{\lambda_{1}}-1}{\sqrt{\lambda_{1}}+1}\right]-1=0 .
$$

After substituting $D$ and multiplying throughout by the common denominator

$$
s_{22} s_{66}\left(\lambda_{1}-\lambda_{2}\right)\left(\sqrt{\lambda_{1}}+1\right)\left(\sqrt{\lambda_{2}}+1\right)
$$

we have

$$
\begin{gathered}
2\left[A_{1}^{2} \sqrt{\lambda_{2}}\left(\sqrt{\lambda_{1}}+1\right)-A_{2}^{2} \sqrt{\lambda_{1}}\left(\sqrt{\lambda_{2}}+1\right)-A_{1} A_{2}\left(\sqrt{\lambda_{1}}-\sqrt{\lambda_{2}}\right)\right]- \\
s_{66}\left[A_{1}\left(\sqrt{\lambda_{1}}+1\right)\left(1-\sqrt{\lambda_{2}}\right)-A_{2}\left(\sqrt{\lambda_{2}}+1\right)\left(1-\sqrt{\lambda_{1}}\right)\right]- \\
\quad s_{22} s_{66}\left(\lambda_{1}-\lambda_{2}\right)\left(\sqrt{\lambda_{1}}+1\right)\left(\sqrt{\lambda_{2}}+1\right)=0 .
\end{gathered}
$$


If we make use of equation (9) and perform some transformations we find

$$
\begin{aligned}
& \left(s_{12}-\lambda_{1} s_{22}\right)\left(\sqrt{\lambda_{1}}+1\right)\left[2\left(s_{12}-\lambda_{1} s_{22}\right) \sqrt{\lambda_{2}}-s_{66}\left(1-\sqrt{\lambda_{2}}\right)\right]- \\
& \left(s_{12}-\lambda_{2} s_{22}\right)\left(\sqrt{\lambda_{2}}+1\right)\left[2\left(s_{12}-\lambda_{2} s_{22}\right) \sqrt{\lambda_{1}}+s_{66}\left(\sqrt{\lambda_{1}}-1\right)\right]+2\left(s_{12}-\lambda_{1} s_{22}\right)\left(s_{12}-\lambda_{2} s_{22}\right)\left(\sqrt{\lambda_{1}}-\sqrt{\lambda_{2}}\right)- \\
& s_{22} s_{66}\left(\lambda_{1}-\lambda_{2}\right)\left(\sqrt{\lambda_{1}}+1\right)\left(\sqrt{\lambda_{2}}+1\right)=0 .
\end{aligned}
$$

If we factor the above expression we have

$$
\left(\sqrt{\lambda_{1}}-\sqrt{\lambda_{2}}\right)\left[s_{12}+s_{22} \sqrt{\lambda_{1}} \sqrt{\lambda_{2}}\left(1+\sqrt{\lambda_{1}}+\sqrt{\lambda_{2}}\right)\right]\left[s_{22}\left(\lambda_{1}+\lambda_{2}\right)-2 s_{12}-s_{66}\right]=0 .
$$

The first factor is obviously different from zero. Hence we should investigate the other two. We shall start with the last one:

$$
s_{22}\left(\lambda_{1}+\lambda_{2}\right)-2 s_{12}-s_{66}=-2 s_{12}-s_{66}+s_{22} \frac{\left(2 s_{12}+s_{66}\right)}{s_{22}}=-2 s_{12}-s_{66}+2 s_{12}+s_{66}=0 .
$$

Since the above expression is zero equation $38 \mathrm{a} / 2$ is truly satisfied.

B.3. The third equation to consider is $33 \mathrm{a}]_{3}$. If we substitute the integrals $\stackrel{(31}{I}_{11}, \stackrel{(34)}{I}_{11}$ and $\stackrel{(41)}{I}_{11}-$ see equations $(34 \mathrm{a}$, , 34d) and (36d) - we obtain

$$
\begin{array}{r}
\frac{2 \pi D}{s_{12}^{2}-s_{11} s_{22}}\left[s_{12}\left(A_{1}^{2} \frac{\lambda_{2}}{\sqrt{\lambda_{2}}+1}-A_{2}^{2} \frac{\lambda_{1}}{\sqrt{\lambda_{1}}+1}\right)+s_{11} A_{1} A_{2} \frac{\sqrt{\lambda_{1}}-\sqrt{\lambda_{2}}}{\left(\sqrt{\lambda_{1}}+1\right)\left(\sqrt{\lambda_{2}}+1\right)}\right]+ \\
2 \pi D\left(\frac{\lambda_{1} A_{2}}{\left(\sqrt{\lambda_{1}}+1\right)}-\frac{\lambda_{2} A_{1}}{\left(\sqrt{\lambda_{2}}+1\right)}\right)=0 .
\end{array}
$$

Let us multiply throughout by the common denominator $\left(s_{12}^{2}-s_{11} s_{22}\right)\left(\sqrt{\lambda_{2}}+1\right)\left(\sqrt{\lambda_{1}}+1\right)$ and substitute $D$. We have

$$
\begin{aligned}
& s_{12}\left[A_{1}^{2} \lambda_{2}\left(\sqrt{\lambda_{1}}+1\right)-A_{2}^{2} \lambda_{1}\left(\sqrt{\lambda_{2}}+1\right)\right]+s_{11} A_{1} A_{2}\left(\sqrt{\lambda_{1}}-\sqrt{\lambda_{2}}\right)+ \\
& \quad\left(s_{12}^{2}-s_{11} s_{22}\right)\left[A_{2} \lambda_{1}\left(\sqrt{\lambda_{2}}+1\right)-A_{1} \lambda_{2}\left(\sqrt{\lambda_{1}}+1\right)\right]=0 .
\end{aligned}
$$

If we make use of equations 9 we find

$$
\begin{aligned}
& s_{12}\left(s_{12}-\lambda_{1} s_{22}\right)^{2} \lambda_{2}\left(\sqrt{\lambda_{1}}+1\right)-s_{12}\left(s_{12}-\lambda_{2} s_{22}\right)^{2} \lambda_{1}\left(\sqrt{\lambda_{2}}+1\right)+ \\
& s_{11}\left(s_{12}-\lambda_{1} s_{22}\right)\left(s_{12}-\lambda_{2} s_{22}\right)\left(\sqrt{\lambda_{1}}-\sqrt{\lambda_{2}}\right)+\left(s_{12}^{2}-s_{11} s_{22}\right)\left(s_{12}-\lambda_{2} s_{22}\right) \lambda_{1}\left(\sqrt{\lambda_{2}}+1\right)- \\
& \quad\left(s_{12}^{2}-s_{11} s_{22}\right)\left(s_{12}-\lambda_{1} s_{22}\right) \lambda_{2}\left(\sqrt{\lambda_{1}}+1\right)=0 .
\end{aligned}
$$

After factoring the above expression we obtain the product:

$$
\left(\sqrt{\lambda_{1}}-\sqrt{\lambda_{2}}\right)\left[-s_{12}+s_{22}\left(\lambda_{1}+\lambda_{2}+\sqrt{\lambda_{1}}+\sqrt{\lambda_{2}}+\sqrt{\lambda_{1}} \sqrt{\lambda_{2}}\right)\right]\left(\lambda_{1} \lambda_{2} s_{22}-s_{11}\right)=0
$$

The first factor is apparently not equal to zero. Therefore we have to consider the other two. The last one clearly vanishes:

$$
\left(-s_{11}+\lambda_{1} \lambda_{2} s_{22}\right)=\left(-s_{11}+\frac{s_{11}}{s_{22}} s_{22}\right)=-s_{11}+s_{11}=0 .
$$

Consequently equation $38 \mathrm{a} 3$ is also satisfied.

\section{Proof of equation $38 \mathrm{~b}$}

C.1. Third, consider equation $38 \mathrm{~b}{ }_{1}$. Upon substitution of the integrals $\stackrel{(31)}{I}_{22}, \stackrel{(34)}{I_{22}}$ and $\stackrel{(41)}{I}_{22}$ - see equations $34 \mathrm{e}$, $34 \mathrm{~h}$ and $36 \mathrm{e}$ - we have

$$
\begin{aligned}
\frac{2 \pi D}{s_{12}^{2}-s_{11} s_{22}}\left[\frac{s_{22} A_{1} A_{2}\left(\sqrt{\lambda_{1}}-\sqrt{\lambda_{2}}\right)}{\left(\sqrt{\lambda_{1}}+1\right)\left(\sqrt{\lambda_{2}}+1\right)}+s_{21}\left(\frac{A_{1}^{2}}{\sqrt{\lambda_{1}}} \frac{1}{\sqrt{\lambda_{1}}+1}-\frac{A_{2}^{2}}{\sqrt{\lambda_{2}}} \frac{1}{\sqrt{\lambda_{2}}+1}\right)\right]- \\
2 \pi D\left(\frac{A_{1}}{\sqrt{\lambda_{1}}\left(\sqrt{\lambda_{1}}+1\right)}-\frac{A_{2}}{\sqrt{\lambda_{2}}\left(\sqrt{\lambda_{2}}+1\right)}\right)=0 .
\end{aligned}
$$


After multiplying throughout by the common denominator

$$
\left(s_{12}^{2}-s_{11} s_{22}\right) \sqrt{\lambda_{1}} \sqrt{\lambda_{2}}\left(\sqrt{\lambda_{1}}+1\right)\left(\sqrt{\lambda_{2}}+1\right)
$$

and making use of equations 9 we obtain

$$
\begin{aligned}
& \left(\sqrt{\lambda_{1}}-\sqrt{\lambda_{2}}\right)\left[s_{12}^{3}\left(\sqrt{\lambda_{1}}+\sqrt{\lambda_{2}}\right)-s_{12}^{2} s_{21}+s_{12}^{3}+\lambda_{1}^{\frac{3}{2}} \lambda_{2}^{\frac{3}{2}} s_{22}^{3}-\sqrt{\lambda_{1}} s_{12}^{2} s_{21}-\right. \\
& -\sqrt{\lambda_{2}} s_{12}^{2} s_{21}--s_{11} s_{12} s_{22}-\sqrt{\lambda_{1}} \sqrt{\lambda_{2}} s_{11} s_{22}^{2}+\underbrace{\sqrt{\lambda_{1}} \lambda{ }_{2}^{\frac{3}{2}} s_{21} s_{22}^{2}-\sqrt{\lambda_{1}} \lambda_{2}^{\frac{3}{2}} s_{12} s_{22}^{2}}+ \\
& \underbrace{\lambda_{1}^{\frac{3}{2}} \sqrt{\lambda_{2}} s_{21} s_{22}^{2}-\lambda_{1}^{\frac{3}{2}} \sqrt{\lambda_{2}} s_{12} s_{22}^{2}}+\lambda_{1} \lambda_{2} s_{21} s_{22}^{2}-\sqrt{\lambda_{1}} s_{11} s_{12} s_{22}-\sqrt{\lambda_{2}} s_{11} s_{12} s_{22}+ \\
& +\lambda_{1} \lambda_{2}^{\frac{3}{2}} s_{21} s_{22}^{2}+\lambda_{1}^{\frac{3}{2}} \lambda_{2} s_{21} s_{22}^{2}+\underbrace{2 \sqrt{\lambda_{1}} \sqrt{\lambda_{2}} s_{12}^{2} s_{22}-2 \sqrt{\lambda_{1}} \sqrt{\lambda_{2}} s_{12}^{2} s_{22}}]=0 .
\end{aligned}
$$

Let us divide throughout by $\sqrt{\lambda_{1}}-\sqrt{\lambda_{2}}$ and cancel the terms braced. If, in addition, we take into account that

$$
-s_{12}^{2} s_{21}+s_{12}^{3}=-s_{12}^{3}+s_{21}^{3}=0
$$

we shall have

$$
\begin{aligned}
&\left(\sqrt{\lambda_{1}} s_{12}^{3}+\sqrt{\lambda_{2}} s_{12}^{3}\right.+\lambda_{1}^{\frac{3}{2}} \lambda_{2}^{\frac{3}{2}} s_{22}^{3}-\sqrt{\lambda_{1}} s_{12}^{2} s_{21}- \\
&-\sqrt{\lambda_{2}} s_{12}^{2} s_{21}-s_{11} s_{12} s_{22}-\sqrt{\lambda_{1}} \sqrt{\lambda_{2}} s_{11} s_{22}^{2}+2 \sqrt{\lambda_{1}} \sqrt{\lambda_{2}} s_{12}^{2} s_{22}-\sqrt{\lambda_{1}} \lambda_{2}^{\frac{3}{2}} s_{12} s_{22}^{2}+ \\
&+\sqrt{\lambda_{1}} \lambda_{2}^{\frac{3}{2}} s_{21} s_{22}^{2}-\lambda_{1}^{\frac{3}{2}} \sqrt{\lambda_{2}} s_{12} s_{22}^{2}+\lambda_{1}^{\frac{3}{2}} \sqrt{\lambda_{2}} s_{21} s_{22}^{2}+\lambda_{1} \lambda_{2} s_{21} s_{22}^{2}-\sqrt{\lambda_{1}} s_{11} s_{12} s_{22}- \\
&\left.\quad-\sqrt{\lambda_{2}} s_{11} s_{12} s_{22}+\lambda_{1} \lambda_{2}^{\frac{3}{2}} s_{21} s_{22}^{2}+\lambda_{1}^{\frac{3}{2}} \lambda_{2} s_{21} s_{22}^{2}-2 \sqrt{\lambda_{1}} \sqrt{\lambda_{2}} s_{12} s_{21} s_{22}\right)=0 \\
& \quad
\end{aligned}
$$

in which

$$
\lambda_{1}^{\frac{3}{2}} \lambda_{2}^{\frac{3}{2}} s_{22}^{3}-s_{11} s_{12} s_{22}-\sqrt{\lambda_{1}} \sqrt{\lambda_{2}} s_{11} s_{22}^{2}+\lambda_{1} \lambda_{2} s_{21} s_{22}^{2}-\sqrt{\lambda_{1}} s_{11} s_{12} s_{22}-\sqrt{\lambda_{2}} s_{11} s_{12} s_{22}+\lambda_{1} \lambda_{2}^{\frac{3}{2}} s_{21} s_{22}^{2}+\lambda_{1}^{\frac{3}{2}} \lambda_{2} s_{21} s_{22}^{2}=0
$$

where

$$
\begin{aligned}
& \lambda_{1} \lambda_{2}^{\frac{3}{2}} s_{21} s_{22}^{2}+\lambda_{1}^{\frac{3}{2}} \lambda_{2} s_{21} s_{22}^{2}-\sqrt{\lambda_{1}} s_{11} s_{12} s_{22}-\sqrt{\lambda_{2}} s_{11} s_{12} s_{22}= \\
& =s_{21} s_{22}^{2} \lambda_{1} \lambda_{2}\left(\sqrt{\lambda_{1}}+\sqrt{\lambda_{2}}\right)-s_{11} s_{12} s_{22}\left(\sqrt{\lambda_{1}}+\sqrt{\lambda_{2}}\right)=s_{21} s_{22}^{2} \frac{s_{11}}{s_{22}}\left(\sqrt{\lambda_{1}}+\sqrt{\lambda_{2}}\right)-s_{11} s_{12} s_{22}\left(\sqrt{\lambda_{1}}+\sqrt{\lambda_{2}}\right)= \\
& =s_{11} s_{12} s_{22}\left(\sqrt{\lambda_{1}}+\sqrt{\lambda_{2}}\right)-s_{11} s_{12} s_{22}\left(\sqrt{\lambda_{1}}+\sqrt{\lambda_{2}}\right)=0 .
\end{aligned}
$$

Therefore

$$
\begin{aligned}
& \lambda_{1}^{\frac{3}{2}} \lambda_{2}^{\frac{3}{2}} s_{22}^{3}+\lambda_{1} \lambda_{2} s_{21} s_{22}^{2}-s_{11} s_{12} s_{22}-\sqrt{\lambda_{1}} \sqrt{\lambda_{2}} s_{11} s_{22}^{2}= \\
& =s_{22}^{2} \lambda_{1} \lambda_{2}\left(s_{22} \sqrt{\lambda_{1}} \sqrt{\lambda_{2}}+s_{21}\right)-s_{12} s_{22}\left(s_{11}+s_{22} \sqrt{\lambda_{1}} \sqrt{\lambda_{2}}\right)=s_{22}^{2} \frac{s_{11}}{s_{22}}\left(s_{22} \sqrt{\lambda_{1}} \sqrt{\lambda_{2}}+s_{21}-s_{12} s_{22}\left(s_{11}+s_{22} \sqrt{\lambda_{1}} \sqrt{\lambda_{2}}\right)=\right. \\
& =s_{11} s_{22}^{2} \sqrt{\lambda_{1}} \sqrt{\lambda_{2}}+s_{11} s_{12} s_{22}-s_{11} s_{12} s_{22}-s_{11} s_{22}^{2} \sqrt{\lambda_{1}} \sqrt{\lambda_{2}}=0 . \\
& \text { (32) (33) (42) (43) }
\end{aligned}
$$

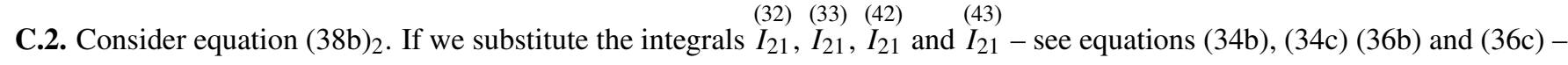
and perform some manipulations we obtain

$$
\begin{aligned}
& 2 \pi D \frac{2}{s_{66}}\left[A_{1}^{2} \frac{1}{\sqrt{\lambda_{1}}+1}-A_{2}^{2} \frac{1}{\sqrt{\lambda_{2}}+1}-A_{1} A_{2} \frac{\sqrt{\lambda_{1}}-\sqrt{\lambda_{2}}}{\left(\sqrt{\lambda_{1}}+1\right)\left(\sqrt{\lambda_{2}}+1\right)}\right]- \\
& -2 \pi D\left(\frac{A_{2}}{\left(\sqrt{\lambda_{2}}+1\right)}-\frac{A_{1}}{\left(\sqrt{\lambda_{1}}+1\right)}\right)-2 \pi D\left(A_{1} \frac{\sqrt{\lambda_{1}}}{\sqrt{\lambda_{1}}+1}-A_{2} \frac{\sqrt{\lambda_{2}}}{\sqrt{\lambda_{2}}+1}\right)=1 \text {. }
\end{aligned}
$$


Let us multiply throughout by the common denominator $s_{66}\left(\sqrt{\lambda_{1}}+1\right)\left(\sqrt{\lambda_{2}}+1\right)$ and make some manipulations. In this way we have

$$
\begin{aligned}
& 2\left[A_{1}^{2}\left(\sqrt{\lambda_{2}}+1\right)\right.\left.-A_{2}^{2}\left(\sqrt{\lambda_{1}}+1\right)\right]-2 A_{1} A_{2}\left(\sqrt{\lambda_{1}}-\sqrt{\lambda_{2}}\right)- \\
&-s_{66}\left[A_{2}\left(\sqrt{\lambda_{1}}+1\right)-A_{1}\left(\sqrt{\lambda_{2}}+1\right)\right]-s_{66}\left[A_{1} \sqrt{\lambda_{1}}\left(\sqrt{\lambda_{2}}+1\right)-A_{2} \sqrt{\lambda_{2}}\left(\sqrt{\lambda_{1}}+1\right)\right]- \\
&-\left(\lambda_{1}-\lambda_{2}\right) s_{22} s_{66}\left(\sqrt{\lambda_{1}}+1\right)\left(\sqrt{\lambda_{2}}+1\right)=0 .
\end{aligned}
$$

If simplify 68 then we have

$$
\begin{aligned}
& 2\left(s_{12}-\lambda_{1} s_{22}\right)^{2}\left(\sqrt{\lambda_{2}}+1\right)-2\left(s_{12}-\lambda_{2} s_{22}\right)^{2}\left(\sqrt{\lambda_{1}}+1\right)- \\
& -2\left(s_{12}-\lambda_{1} s_{22}\right)\left(s_{12}-\lambda_{2} s_{22}\right)\left(\sqrt{\lambda_{1}}-\sqrt{\lambda_{2}}\right)+s_{66}\left(s_{12}-\lambda_{1} s_{22}\right)\left(\sqrt{\lambda_{2}}+1\right)\left(1-\sqrt{\lambda_{1}}\right)+ \\
& \quad+s_{66}\left(s_{12}-\lambda_{2} s_{22}\right)\left(\sqrt{\lambda_{1}}+1\right)\left(\sqrt{\lambda_{2}}-1\right)-\left(\lambda_{1}-\lambda_{2}\right) s_{22} s_{66}\left(\sqrt{\lambda_{1}}+1\right)\left(\sqrt{\lambda_{2}}+1\right)=0 .
\end{aligned}
$$

If we factoring this expression we shall get

$$
\left(\sqrt{\lambda_{1}}-\sqrt{\lambda_{2}}\right)\left(s_{12}+\sqrt{\lambda_{1}} s_{22}+\sqrt{\lambda_{2}} s_{22}+\sqrt{\lambda_{1}} \sqrt{\lambda_{2}} s_{22}\right)\left(-2 s_{12}-s_{66}+\lambda_{1} s_{22}+\lambda_{2} s_{22}\right)=0 .
$$

The first factor is evidently different from zero. Hence we have to investigate the other two. We shall start with the last one

$$
\left(-2 s_{12}-s_{66}+\lambda_{1} s_{22}+\lambda_{2} s_{22}\right)=-2 s_{12}-s_{66}+s_{22}\left(\lambda_{1}+\lambda_{2}\right)=-2 s_{12}-s_{66}+s_{22} \frac{2 s_{12}+s_{66}}{s_{22}}=-2 s_{12}-s_{66}+2 s_{12}+s_{66}=0 .
$$

C.3. Third consider equation $38 \mathrm{~b}, 3$. Upon substitution of the integrals $\stackrel{(31)}{I}_{22}, \stackrel{(34)}{I}_{22}$ and $\stackrel{(41)}{I}_{22}$ - see equations $34 \mathrm{e}$, 34h and $36 \mathrm{e}$ we have

$$
\begin{aligned}
&-\frac{2 \pi D}{s_{12}^{2}-s_{11} s_{22}}\left[\frac{s_{12} A_{1} A_{2}\left(\sqrt{\lambda_{1}}-\sqrt{\lambda_{2}}\right)}{\left(\sqrt{\lambda_{1}}+1\right)\left(\sqrt{\lambda_{2}}+1\right)}+\left(\frac{A_{1}^{2}}{\sqrt{\lambda_{1}}\left(\sqrt{\lambda_{1}}+1\right)}-\frac{A_{2}^{2}}{\sqrt{\lambda_{2}}\left(\sqrt{\lambda_{2}}+1\right)}\right)\right]- \\
& 2 \pi D\left(\frac{A_{1}}{\sqrt{\lambda_{1}}+1}-\frac{A_{2}}{\sqrt{\lambda_{2}}+1}\right)=1 .
\end{aligned}
$$

Then multiplying throughout by the common denominator

$$
\left(s_{12}^{2}-s_{11} s_{22}\right) \sqrt{\lambda_{1}} \sqrt{\lambda_{2}}\left(\sqrt{\lambda_{1}}+1\right)\left(\sqrt{\lambda_{2}}+1\right)
$$

and making some manipulations we obtain

$$
\begin{array}{r}
-s_{12} A_{1} A_{2} \sqrt{\lambda_{1} \lambda_{2}}\left(\sqrt{\lambda_{1}}-\sqrt{\lambda_{2}}\right)-s_{11}\left[A_{1}^{2} \sqrt{\lambda_{2}}\left(\sqrt{\lambda_{2}}+1\right)-A_{2}^{2} \sqrt{\lambda_{1}}\left(\sqrt{\lambda_{1}}+1\right)\right]- \\
-\left(s_{12}^{2}-s_{11} s_{22}\right) \sqrt{\lambda_{1} \lambda_{2}}\left[A_{1}\left(\sqrt{\lambda_{2}}+1\right)-A_{2}\left(\sqrt{\lambda_{1}}+1\right)\right]- \\
-\left(\lambda_{1}-\lambda_{2}\right) s_{22}\left(s_{12}^{2}-s_{11} s_{22}\right) \sqrt{\lambda_{1} \lambda_{2}}\left(\sqrt{\lambda_{1}}+1\right)\left(\sqrt{\lambda_{2}}+1\right)=0 .
\end{array}
$$

If we substitute equation 9 , we have

$$
\begin{aligned}
& s_{11} s_{12} \lambda_{1}-s_{11} s_{12} \lambda_{2}+s_{12} s_{22} \lambda_{1} \lambda_{2}^{2}-s_{12} s_{22} \lambda_{1}^{2} \lambda_{2}+s_{11} s_{12} \sqrt{\lambda_{1}}-s_{11} s_{12} \sqrt{\lambda_{2}}+ \\
& +s_{11} s_{22} \lambda_{1} \sqrt{\lambda_{2}}-s_{11} s_{22} \sqrt{\lambda_{1}} \lambda_{2}+s_{12} s_{22} \lambda_{1} \lambda_{2}^{\frac{3}{2}}-s_{12} s_{22} \lambda_{1}^{\frac{3}{2}} \lambda_{2}-s_{22}^{2} \lambda_{1}^{2} \lambda_{2}^{\frac{3}{2}}+s_{22}^{2} \lambda_{1}^{\frac{3}{2}} \lambda_{2}^{2}=0 .
\end{aligned}
$$

After factoring this equations we shall find

$$
\left(\sqrt{\lambda_{1}}-\sqrt{\lambda_{2}}\right)\left(s_{12}+\sqrt{\lambda_{1}} s_{12}+\sqrt{\lambda_{2}} s_{12}+\sqrt{\lambda_{1}} \sqrt{\lambda_{2}} s_{22}\right)\left(s_{11}-\lambda_{1} \lambda_{2} s_{22}\right)=0 .
$$

One can check easily that the third factor is zero:

$$
\left(s_{11}-\lambda_{1} \lambda_{2} s_{22}\right)=\left(s_{11}-\frac{s_{11}}{s_{22}} s_{22}\right)=s_{11}-s_{11}=0 .
$$

Consequently $38 \mathrm{~b}]_{3}$ is fulfilled. 


\section{Formulae for strains}

Making use of equations (13), (14) and (40) from the kinematic equations (1) we obtain

$$
e_{\alpha \beta}=\oint_{\mathcal{L}_{o}} t_{\lambda}\left(M_{\circ}\right) \mathcal{D}_{\lambda \alpha \beta}\left(M_{\circ}, Q\right) \mathrm{d} s_{M_{\circ}}-\oint_{\mathcal{L}_{o}} u_{\lambda}\left(M_{\circ}\right) \mathcal{S}_{\lambda \alpha \beta}\left(M_{\circ}, Q\right) \mathrm{d} s_{M_{\circ}} \quad Q \in A_{e}
$$

where

$$
\begin{array}{llrl}
\mathcal{D}_{\lambda 11} & =U_{\lambda 1} \partial_{1}, & \mathcal{S}_{\lambda 11} & =T_{\lambda 1} \partial_{1}, \\
\mathcal{D}_{\lambda 12} & =\frac{1}{2}\left(U_{\lambda 2} \partial_{1}+U_{\lambda 1} \partial_{2}\right)=\mathcal{D}_{\lambda 21}, & \mathcal{S}_{\lambda 12} & =\frac{1}{2}\left(T_{\lambda 2} \partial_{1}+T_{\lambda 1} \partial_{2}\right)=\mathcal{D}_{\lambda 21}, \\
\mathcal{D}_{\lambda 22} & =U_{\lambda 2} \partial_{2}, & \mathcal{S}_{\lambda 22} & =T_{\lambda 2} \partial_{2} .
\end{array}
$$

If we introduce the notations

$$
\begin{aligned}
& b_{1}=\frac{\sqrt{\lambda_{1}}}{\rho_{1}^{2}}, \quad b_{2}=\frac{\sqrt{\lambda_{2}}}{\rho_{2}^{2}}, \quad c=\sqrt{\lambda_{1}} \sqrt{\lambda_{2}} r_{1}^{2}+r_{2}^{2}, \quad d=\left(\sqrt{\lambda_{1}}-\sqrt{\lambda_{2}}\right) r_{2}, \\
& k_{1}=\frac{\lambda_{1}^{\frac{3}{2}} A_{1}}{\rho_{1}^{2}}, \quad k_{2}=\frac{\lambda_{2}^{\frac{3}{2}} A_{2}}{\rho_{2}^{2}}, \quad f_{1}=\frac{A_{1}}{\left(\lambda_{1}^{\frac{3}{2}} r_{1}^{2}+r_{2}^{2}\right)^{2}}, \quad f_{2}=\frac{A_{2}}{\left(\lambda_{2}^{\frac{3}{2}} r_{1}^{2}+r_{2}^{2}\right)^{2}}
\end{aligned}
$$

for the derivatives in equation 79 we have

$$
\begin{aligned}
& \partial_{1} U_{11}=D\left(b_{2} \lambda_{2} A_{1}^{2} r_{1}-b_{1} \lambda_{1} A_{2}^{2} r_{1}\right), \\
& \partial_{1} U_{12}=\frac{D A_{1} A_{2}}{1+d^{2} r_{1}^{2}}\left(2 \sqrt{\lambda_{1}} \sqrt{\lambda_{2}} d r_{1}^{2}-c d\right)=\partial_{1} U_{21}, \\
& \partial_{1} U_{22}=D\left(b_{1} A_{1}^{2} r_{1}-b_{2} A_{2}^{2} r_{1}\right), \\
& \partial_{2} U_{11}=D\left(b_{2} A_{1}^{2} r_{2}-b_{1} A_{2}^{2} r_{2}\right), \\
& \partial_{2} U_{12}=\frac{D A_{1} A_{2}}{1+d^{2} r_{1}^{2}}\left[2 d r_{1} r_{2}-c\left(\sqrt{\lambda_{1}}-\sqrt{\lambda_{2}}\right) r_{1}\right]=\partial_{2} U_{21}, \\
& \partial_{2} U_{22}=-D\left(\frac{A_{2}^{2} r_{2}}{\rho_{2}^{2} \sqrt{\lambda_{2}}}-\frac{A_{1}^{2} r_{2}}{\rho_{1}^{2} \sqrt{\lambda_{1}}}\right)
\end{aligned}
$$

and

$$
\begin{aligned}
& \partial_{1} T_{11}=D {\left[\frac{2 b_{2} \lambda_{2} A_{1} r_{1}}{\rho_{2}^{2}}-\frac{2 b_{1} \lambda_{1} A_{2} r_{1}}{\rho_{1}^{2}}\right]\left(r_{1} n_{1}+r_{2} n_{2}\right)-D\left[b_{2} A_{1}-b_{1} A_{2}\right] n_{1}, } \\
& \partial_{1} T_{12}=D {\left[\frac{2 b_{1} \lambda_{1} A_{1} r_{1}}{\rho_{1}^{2}}-\frac{2 b_{2} \lambda_{2} A_{2} r_{1}}{\rho_{2}^{2}}\right] r_{1} n_{2}-D\left[b_{1} A_{1}-b_{2} A_{2}\right] n_{2}-D\left[2 f_{1} \lambda_{1} r_{1}-2 f_{2} \lambda_{2} r_{1}\right] r_{2} n_{1}, } \\
& \partial_{1} T_{21}=D {\left[\frac{2 b_{1} \lambda_{1}^{2} A_{2} r_{1}}{\rho_{1}^{2}}-\frac{2 b_{1} \lambda_{2}^{2} A_{1} r_{1}}{\rho_{2}^{2}}\right] r_{1} n_{2}-D\left[b_{1} \lambda_{1} A_{2}-b_{2} \lambda_{2} A_{1}\right] n_{2}-D\left[\frac{2 b_{1} \lambda_{1} A_{2} r_{1}}{\rho_{1}^{2}}-\frac{\left.2 b_{2} \lambda_{2} A_{1} r_{1}\right]}{\rho_{2}^{2}}\right] r_{2} n_{1}, } \\
& \partial_{1} T_{22}=D\left[\frac{2 b_{1} \lambda_{1} A_{1} r_{1}}{\rho_{1}^{2}}-\frac{2 b_{2} \lambda_{2} A_{2} r_{1}}{\rho_{2}^{2}}\right]\left(r_{1} n_{1}+r_{2} n_{2}\right)-D\left[b_{1} A_{1}-b_{2} A_{2}\right] n_{1}, \\
& \partial_{2} T_{11}=D\left[\frac{b_{2} A_{1} 2 r_{2}}{\rho_{2}^{2}}-\frac{b_{1} A_{2} 2 r_{2}}{\rho_{1}^{2}}\right]\left(r_{1} n_{1}+r_{2} n_{2}\right)-D\left[b_{2} A_{1}-b_{1} A_{2}\right] n_{2}, \\
& \partial_{2} T_{12}=D\left[\frac{b_{1} A_{1} 2 r_{2}}{\rho_{1}^{2}}-\frac{b_{2} A_{2} 2 r_{2}}{\rho_{2}^{2}}\right] r_{1} n_{2}-D\left[2 f_{1} r_{2}-2 f_{2} r_{2}\right] r_{2} n_{1}+D\left[f_{1}-f_{2}\right] n_{1}, \\
& \partial_{2} T_{21}=D\left[\frac{b_{1} \lambda_{1} A_{2} 2 r_{2}}{\rho_{1}^{2}}-\frac{b_{2} \lambda_{2} A_{1} 2 r_{2}}{\rho_{2}^{2}}\right] r_{1} n_{2}-D\left[\frac{b_{1} A_{2} 2 r_{2}}{\rho_{1}^{2}}-\frac{b_{2} A_{1} 2 r_{2}}{\rho_{2}^{2}}\right] r_{2} n_{1}+D\left[b_{1} A_{2}-b_{2} A_{1}\right] n_{1}, \\
& \partial_{2} T_{22}=D\left[\frac{b_{1} A_{1} 2 r_{2}}{\rho_{1}^{2}}-\frac{b_{2} A_{2} 2 r_{2}}{\rho_{2}^{2}}\right]\left(r_{1} n_{1}+r_{2} n_{2}\right)-D\left[b_{1} A_{1}-b_{2} A_{2}\right] n_{2} .
\end{aligned}
$$

